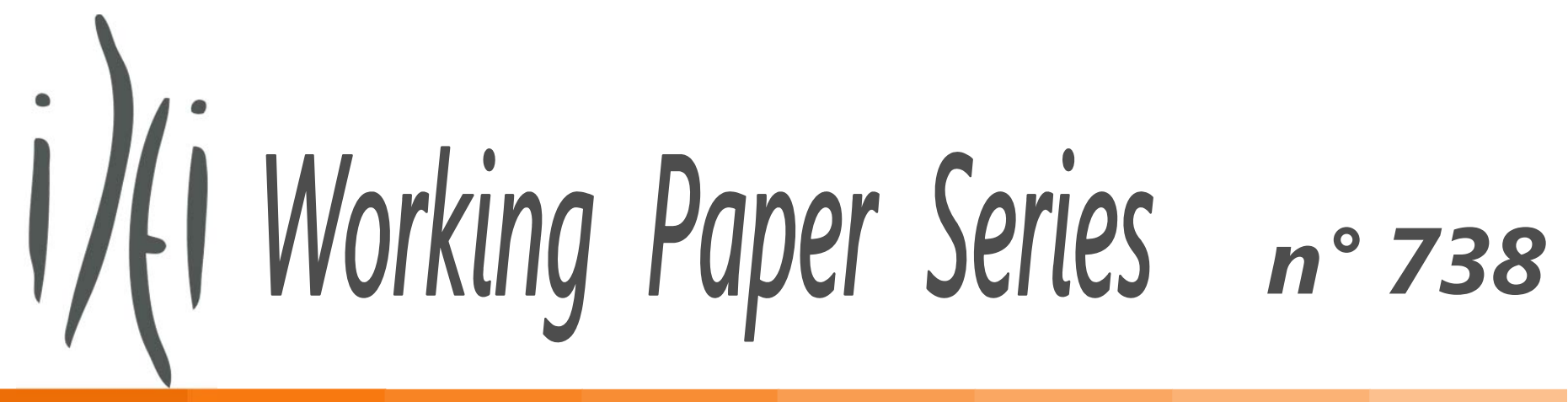

August 2012

\title{
« Understanding the Effect of Technology Shocks in SVARs with Long-Run Restrictions »
}

\section{Jérémy Chaudourne, Patrick Fève and Alain Guay}




\title{
Understanding the Effect of Technology Shocks in SVARs with Long-Run Restrictions
}

\author{
Jeremy Chaudourne \\ Université du Québec à Montréal and CIRPÉE \\ Patrick Fève* \\ TSE (University of Toulouse, GREMAQ, IDEI, IUF and Banque de France) \\ Alain Guay \\ Université du Québec à Montréal, CIRPÉE and CIREQ
}

August, 2012

\begin{abstract}
This paper studies the statistical properties of impulse response functions in structural vector autoregressions (SVARs) with a highly persistent variable as hours worked and long-run identifying restrictions. The highly persistent variable is specified as a nearly stationary persistent process. Such process appears particularly well suited to characterized the dynamics of hours worked because it implies a unit root in finite sample but is asymptotically stationary and persistent. This is typically the case for per capita hours worked which are included in SVARs. Theoretical results derived from this specification allow to explain most of the empirical findings from SVARs which include U.S. hours worked. Simulation experiments from an estimated DSGE model confirm theoretical results.
\end{abstract}

Keywords: SVARs, long-run restrictions, locally nonstationary process, technology shocks, hours worked

JEL Class.: C32, E32

\footnotetext{
*Address: GREMAQ-Université de Toulouse I, manufacture des Tabacs, bât. F, 21 allée de Brienne, 31000 Toulouse. emails: chaudourne.jeremy@courrier.uqam.ca, patrick.feve@univ-tlse1.fr and guay.alain@uqam.ca. We would like to thank J. Fernald, J.P. Florens, A. Marcet and V. Ramey for valuable remarks and suggestions. This paper has benefited from helpful discussions during presentations at various seminars and conferences. The traditional disclaimer applies. The views expressed herein are those of the authors and not necessary those of the Banque de France.
} 


\section{Introduction}

The dynamic response of hours worked to a permanent technology shock identified from SVARs is a controversial issue. The specification of hours in SVARs and the measurement of the productivity appear crucial in the explanation of apparent conflictual results (see e.g. Galí, 1999, Francis and Ramey, 2005, Basu, Fernald and Kimball, 2006, Christiano, Eichenbaum and Vigfusson, 2004a, 2004b, Chari, Kehoe and McGrattan, 2008 and Fève and Guay, 2009). The aim of this paper is to explain these conflicting results in SVARs using a simple statistical framework with hours worked modelized as a locally nonstationary process. Our key result is that low-frequency movements in hours can contaminate the identification of technology shocks when labor productivity is included in SVARs.

In the first part of the paper, we characterize empirical evidences obtained from SVARs with US data. These findings will then be used as basic facts to be explained by our statistical framework. These results also echo those obtained in Galí (1999), Francis and Ramey (2005), Basu, Fernald and Kimball (2006), Christiano, Eichenbaum and Vigfusson (2004a) and (2004b). Using different productivity measures, we compute the dynamic responses of hours worked after a technology improvement. The main results are the following. First, when a "purified" measure of total factor productivity (TFP) constructed by Basu, Fernald and Kimball (2006) is included in SVARs, the specification of hours does not matter as we obtain almost the same dynamic responses: hours decrease in short-run and thus display a positive hump-shaped pattern. Second, when labor productivity is used, the response of hours with the LSVAR model (hours are specified in level) is positive, but it becomes persistently negative with the DSVAR model (hours are specified in first difference) and both differ from the ones obtained with the "purified" measure of total factor productivity. Third, confidence bands with the LSVAR model are fairly large and thus not informative.

Some existing works have tried to explain conflicting results obtained with LSVAR and DSVAR models. Christiano, Eichenbaum and Vigfusson (2004a) and Gospodinov (2010) establish that the large sampling uncertainty with the LSVAR model can arise from a weak instrument problem when the largest root is unity or near the nonstationary boundary (stationary in small sample but asymptotically nonstationary). A LSVAR model for such process thus leads to an inconsistent estimator of the technology shocks. Using also a nearly non-stationary SVAR, Gospodinov, Maynard and Pesavento (2011) explain that the difference in the empirical impulse responses by a small low-frequency comovements between hours worked and productivity growth which appears 
in the LSVAR specification but disappears in the DVAR specification. While a near integrated (or a unit root) process can provide a good statistical approximation in finite sample for a highly persistent variable, as per capita hours worked (see Francis and Ramey, 2005), the maximal number of hours that a person can work in a day is bounded and therefore its stochastic process cannot possess a unit root asymptotically. ${ }^{1}$ No model that takes into account this physical constraint can yield a unit root process for the logarithm of hours (see Chari, Kehoe and McGrattan, 2008, for a discussion). Consequently, the asymptotic nonstationarity for hours cannot be considered as an adequate representation. A characterization of the hours worked as a locally nonstationary but asymptotically stationary persistent process seems more compatible with empirical evidence and theoretical implications. SVAR models with a nearly stationary persistent variable seem to offer a more appropriate representation for studying the properties of the SVAR estimators and the associated dynamic responses in a representation including a highly persistent variable, such as hours worked. The nearly stationary persistent process is also more in connection with equilibrium business cycle models, in which hours worked are stationary but display high serial correlation (see e.g. Erceg, Guerrieri and Gust, 2005, Christiano, Eichenbaum and Vigfusson, 2006, Chari, Kehoe and McGrattan, 2008, Fève and Guay, 2009). ${ }^{2}$

The second part of the paper studies the properties of estimators and impulse response function (IRF) to a permanent shock (i.e. a technology shock), when the nonstationary variable (a TFP measure or the labor productivity) has asymptotically an exact unit root and the other variable (hours worked) follows a nearly stationary persistent process. We show that the estimated responses from the LSVAR model are biased in finite sample if the measure of productivity is contaminated by low frequency movements in hours. However, if the econometrician uses a proper measure of TFP, the bias disappears. We also derive the asymptotic distribution for the structural parameters of the LSVAR model. The estimators are asymptotically consistent, but display a nonstandard limiting distribution. This explains the large confidence interval for the dynamic responses in the LSVAR setup. As for the LSVAR model, the estimated responses obtained from the DSVAR model are biased in finite sample if the productivity measure (i.e. the labor productivity) includes the low-frequency movements in hours. This bias occurs because two shocks seem to have permanent effect on labor productivity in finite sample, violating the long-run identifica-

\footnotetext{
${ }^{1}$ A stationary stochastic representation does not prevent that hours will eventually exceed any positive limit, but the problem is all the more reinforced if they contain a unit root.

${ }^{2}$ Chang, Doh and Schorfheide (2007) consider a DSGE model with nonstationary hours and show that a level specification of hours must be preferred to a difference specification when the model includes real frictions in the form of labor adjustment costs.
} 
tion scheme. This finding explains the huge difference in the estimated IRF between the LSVAR and DSAVR models. When the econometrician uses a "purified" measure of TFP instead of the labor productivity, the DSVAR can yield close to consistent estimates. In this case, the longrun restriction is valid, because the low-frequency movements in hours does not contaminate the measure of productivity. This result explains the empirical findings that show that the LSVAR and DSVAR models yield close dynamic responses of hours when a "purified" measure of TFP is included in the VAR model. Our framework also explains why the dynamic responses from LSVAR and DSVAR with a "purified" measure of TFP differ from the ones identified with the LSVAR and DSVAR using labor productivity. These theoretical results reveal that our statistical framework explains well the three empirical findings discussed earlier from SVARs with U.S. data. As an alternative explanation, Gospodinov, Maynard and Pesavento (2011) propose a SVAR setup with low-frequency comovements. These comovements, that are present in a level representation, disappear in the DSVAR model. While suitable to explain why LSVAR and DSVAR models produce different results, this setup cannot explain why the estimated dynamic responses from LSVAR and DSVAR with labor productivity are biased in small sample as shown using simulations (Chari, Kehoe and McGrattan, 2008 and Fève and Guay, 2010) and differ from the ones with a "purified" measure of $\mathrm{TFP}^{3}$ Our paper also complete theirs by showing that the variable that has the unit root must contain a tiny portion of non-technology shocks in order to obtain accurate estimates of the dynamic responses, as previously emphasized in Chari, Kehoe and McGrattan (2008) and Fève and Guay (2010).

We also performs simulation experiments from an estimated DSGE model in finite sample that illustrate and support our asymptotic results. In particular, we obtain that the low-frequency movements in hours sizeably contribute to hours fluctuations. These movements then contaminate the labor productivity and corrupt the identification scheme. Conversely, when the econometrician can observe the true TFP, both LSVAR and DSVAR models consistently estimate the true response of hours to a technology shocks. As an additional check of our setup, we evaluate the dynamic responses of the productivity measure to a technology shocks. Our simulation experiments support our theoretical findings and show that the estimated responses over-estimate the true ones when

\footnotetext{
${ }^{3}$ Christiano, Eichenbaum and Vigfusson (2006) obtained dynamic responses with a small bias for LSVAR using labor productivity. At the first sight, this could appear at odds with our explanation. However, this result can be explained by the small portion of the labor productivity's variance explained by the non-technology shock thanks to their parameters configuration used to perform their simulations. In fact, for the parameter values used by Christiano, Eichenbaum and Vigfusson (2006), the resulting labor productivity is closed to be an adequate measure of the TFP. This also holds for some results in Fève and Guay (2010) (see Table 2, case with $\rho_{\chi}=.99$ and $\sigma_{\chi} / \sigma_{\chi}=.5$ ) where parameter values are close to the ones used by Christiano, Eichenbaum and Vigfusson (2006).
} 
a contaminated measure of hours (and thus labor productivity) is included in SVARs.

The paper is organized as follows. In a first section, we reports estimated dynamic responses with US data. Section 2 presents the statistical framework and an illustrative economic model to motivate the adopted specification of hours worked. Section 3 analyzes finite sample and asymptotic behaviors of SVARs with a nearly stationary persistent process. Section 4 reports simulation experiments from a DSGE model. The last section concludes. Proofs are reported in Appendix.

\section{Figures with US Data}

In this section, we report some figures on the short-run responses of hours worked in various SVARs estimated with actual US data. SVARs include three different measures of productivity that are then used for long-run identification. In each case, this variable is assumed to have an exact unit root. ${ }^{4}$ The productivity variables considered successively are the Solow residual, a "purified" measure of TFP and the labor productivity. All the productivity measures are specified in logs and in first difference. Data are borrowed from Basu, Fernald and Kimball (2006). The data frequency is annual and covers the sample period 1949-1996. ${ }^{5}$ Our measure of hours worked is the log of non-farm business hours, divided by the population 16 and over. The data used in this empirical analysis are reported in Figure 1. The three measures of productivity display similar business cycle patterns, but the "purified" measure is less volatile than the other two. In addition, the Solow residual and the labor productivity growth are imperfectly correlated with the "purified" measure (the correlation coefficient between the growth rates is 0.31 and 0.57 , respectively). As shown in the right hand side of Figure 1, the log of hours displays persistent fluctuations over the sample period. The estimated autocorrelation function suggests that hours worked display a high serial correlation. We also perform an Augmented Dickey Fuller (ADF) test of unit root. We regress the growth rate of hours on a constant, lagged level and two lags of the first difference. The $\mathrm{ADF}$ test statistic is equal to -1.26 and the null hypothesis of unit root cannot be rejected at $5 \%$. This finding suggests that hours are nonstationary and thus must be specified in first difference in SVARs. However, it is well known that unit root tests have lower power in small sample against stationary alternatives, so hours are specified either in level or in first difference in SVARs.

\footnotetext{
${ }^{4}$ This is confirmed by unit root tests on each measure of productivity. The unit root on the level of each measure is not rejected, whereas the unit root on the first difference is rejected at conventional level.

${ }^{5}$ Fernald (2009) proposes a quarterly version of these technology measures. We do not use these new data, since they do not account for important features (time varying markups and heterogeneity) that are present in the annual frequency data of Basu, Fernald and Kimball (1996).
} 
The four first SVARs (with hours either in level or in first difference) use direct measures of TFP and correspond to the ones adopted by Basu, Fernald and Kimball (2006) and Christiano, Eichenbaum and Vigfusson (2004b). The SVAR model with labor productivity growth and the $\log$ of hours in level is the one adopted by Christiano, Eichenbaum and Evans (2004a), whereas the SVAR with the log of hours in first difference is the one adopted by Galí (1999), Galí and Rabanal (2004) and Francis and Ramey (2005). In all cases, we consider a bivariate VAR model and we impose the long restriction à la Blanchard and Quah (1989) that technology shock is the only variable that can have a permanent effect on the Solow residual, the "purified" measure of TFP and the labor productivity. Following Basu, Fernald and Kimball (2006) and Christiano, Eichenbaum and Evans (2004b), the lag length for each VAR model is two. Results are found to be robust to other choices of the lag length. The confidence interval is then obtained from standard bootstrap techniques with 1000 replications. The results are reported in Figures 2 and 3. For comparison purpose, we set the same scale for each estimated responses. We will consider two sets of dynamic responses. First, we focus on the dynamic responses of hours after a technology improvement. Second, we inspect the response of the three productivity measures to a technology shock.

Let us first consider the dynamic responses of hours, as they constitute a central and debatable empirical issue (see Figure 2). When the Solow residual and the "purified" measure of TFP are included in the SVAR model, the specification of hours (in level or in first difference) does not matter a lot. On impact, hours decrease, but after two years the response becomes persistently positive and displays an hump-shaped pattern. ${ }^{6}$ Interestingly, the discrepancy of IRFs (between the level and the first difference specification) is less pronounced when a "purified" measure of TFP is considered instead of the Solow residual. This finding means that when a proper measure of productivity is available, consistent estimates of the dynamic responses of hours worked can be obtained without wondering what is the proper specification of hours. When the growth rate of labor productivity is included in the SVAR model, things however change dramatically. Indeed, the LSVAR and the DSVAR models predict opposite conclusions about the dynamic responses of hours and for both specifications the dynamic responses differ from the ones obtained by the "purified" measure of TFP. Except on impact, the LSVAR model displays a positive hump-shaped response whereas the DSVAR model implies a persistent decrease in hours (see Galí, 1999, Christiano, Eichenbaum and Evans, 2004a and Francis and Ramey, 2005). Finally, in the case of a level

\footnotetext{
${ }^{6}$ See also Vigfusson, 2004, for similar empirical findings.
} 
specification of hours, the confidence interval is so wide that the estimated IRFs of hours are not significantly different from zero at any horizon. This is especially true when the labor productivity growth is included in the LSVAR model. We also obtain fairly large confidence bands with direct productivity measures. When hours worked are specified in first difference, the confidence interval remains wide but the dynamic responses appear more precisely estimated, especially in the very short run. ${ }^{7}$

Let us now consider the estimated dynamic responses of each productivity measure to a technology improvement. The responses are reported in Figure 3. The responses are comparable for the three productivity measures, because each of them will permanently adjusts in the long-run after a permanent technology shock. The "purified" measure of TFP shifts up almost immediately and the Solow residual after one period. Notice that the specification of hours does not matter, as the dynamic responses with these two measures are very similar. This finding is again in contrast with those obtained from labor productivity. The LSVAR and DSVAR models display different responses in the short-run. The DSVAR model implies a quick adjustment of labor productivity to its new long-run value. With the LSVAR model, the labor productivity adjusts very gradually and persistently. The long-run effect of the technology measure can be directly obtained from the Cholesky decomposition of the long-run covariance matrix of the variables. Results are reported

in Table 1. For each measure, the long effect is almost similar whatever the specification of hours. ${ }^{8}$ Notice however, that the discrepancy is more pronounced in the case of the labor productivity. Again, the "purified" implies the smaller difference and the Solow residual intermediate results. Another important aspect is the sizeable difference between the "purified" measure and the labor productivity. For example, with the DSVAR specification, the long-run effect is $1.39 \%$ with the "purified" measure and $2.32 \%$ with the labor productivity. Using this latter variable yields larger long-run effects of a technology improvement.

\section{The Statistical Framework and an Illustrative Economic Model}

We first present the specification of a nearly stationary persistent process. Second, we connect this statistical representation to the dynamics of hours worked obtained from a standard RBC model.

\footnotetext{
${ }^{7}$ We can also complete these figures by computing the correlation of the technology innovations identified from LSVAR and DSVAR models, for each measure of productivity. The higher correlation is obtained in the case of the "purified" measure of TFP, 0.9860, and the lower is obtained when labor productivity is included in SVARs, 0.9082. The Solow residual provides intermediate results with a correlation of 0.9831 .

${ }^{8}$ The DSVAR model produces larger long-run responses than the LSVAR model with every productivity measure.
} 


\subsection{SVARs with a Nearly Stationary Persistent Process}

In this section we present and study our proposed specification of a highly persistent process. This is obtained by parameterizing it as a nearly stationary persistent process. Phillips (1987) and Chan and Wei (1987), among others, considered nearly unit-root process to investigate the asymptotic power of the unit-root tests under a sequence of local alternatives. Since we are interested by a highly persistent process which is asymptotically stationary, we consider a sequence of local alternatives such that the process is locally nonstationary but asymptotically stationary and persistent.

Let us first introduce this nearly stationary persistent process parametrization with a simple example. For the aim of exposition, suppose the following univariate process:

$$
\begin{aligned}
(1-\rho L) \Delta x_{t} & =u_{t}-\delta_{T} u_{t-1} \\
\delta_{T} & =(1-c / \sqrt{T})
\end{aligned}
$$

with $0<\rho<1, c>0$ and $u_{t}$ is a white noise. As $T$ increasing to infinity and for a high value of $\rho$, this process becomes a stationary persistent process whereas, in finite sample, the process is characterized by an unit root. This process is locally nonstationary but asymptotically stationary and persistent. This characterization of the highly persistent process has two advantages. First, it suitably represents the time series behavior of variables for which usual unit root tests cannot reject the nonstationarity in small sample. Second, although highly persistent, the variable is necessarily characterized by an asymptotic stationary process. Pantula (1991), Perron and Ng (1996) and Ng and Perron (2001) consider a simplified version of this process with $\rho=0$ but to investigate the performance of unit-root tests. ${ }^{9}$ It is important to understand that this specification adopted here must not be interpreted as a literal description of the data but as a device to approximate the behavior of a highly persistent variable in small sample which is necessarily stationary asymptotically.

More generally, we are interested in a bivariate representation $X_{t}=\left(\Delta X_{1 t}, X_{2 t}\right)$ for $t=1, \ldots, T$, where the variable $X_{1 t}$ contains an exact unit root and $X_{2 t}$ is a highly persistent variable. Both variables in the vector $X_{t}$ are asymptotically second order stationary and they admit asymptotically the following Wold decomposition

$$
X_{t}=C(L) \varepsilon_{t},
$$

\footnotetext{
${ }^{9}$ Obviously, the parametrization adopted by these authors is not suitable in our case since such process is asymptotically a white noise.
} 
where $C(L)=\sum_{j=0}^{\infty} C_{j} \varepsilon_{t-j}$ and $C_{0}=I_{2}, \sum_{j=0}^{\infty} C_{j}^{2}<\infty$. $\varepsilon_{t}$ is a vector of white noise processes with $E\left(\varepsilon_{t}\right)=0$ and $E\left(\varepsilon_{t} \varepsilon_{t}^{\prime}\right)=\Sigma$. The deterministic part is omitted to simplify the presentation without altering the results below. Now consider a Structural Moving Average (SMA) representation for $X_{t}$ :

$$
X_{t}=A(L) \eta_{t}
$$

where $A(L)=\sum_{j=0}^{\infty} A_{j}$ and $\eta_{t}=\left(\eta_{1 t}, \eta_{2 t}\right)^{\prime}$ is the vector of orthogonal structural shocks with $E\left(\eta_{t} \eta_{t}^{\prime}\right)=\Omega$ a diagonal matrix. A common identification assumption is $\Omega=I_{2}$, the variance of the structural shocks is then normalized to one.

Given representations (1) and (2), the error terms $\varepsilon_{t}$ from the reduced form are related to the structural error terms $\eta_{t}$ as follows: $\varepsilon_{t}=A_{0} \eta_{t}$ which implies that $\Sigma=A_{0} A_{0}^{\prime}$. The SMA representation is identified through the identification of the relationship matrix $A_{0}$. We focus here on the identification strategy based on long-run restrictions such as proposed by Blanchard and Quah (1989). The identification scheme uses the long-run variance-covariance matrix of the reduced from (1) and the structural form (2) which are related by $C(1) \Sigma C(1)^{\prime}=A(1) A(1)^{\prime}$ and $A_{0}=C(1)^{-1} A(1)$. Typically a lower triangular structure is imposed to the long-run impact matrix $A(1)$ which can be easily obtained using a Choleski decomposition of the long-run variancecovariance matrix $C(1) \Sigma C(1)^{\prime}$. In the case where two variables are included in $X_{t}$, the first structural shock is the only one shock that can have a permanent effect on the first variable.

Now consider for a finite sample of $T$ observations a structural characterization of the highly persistent variable $X_{2 t}$ as a nearly stationary persistent process:

$$
\begin{aligned}
\Delta X_{2 t} & =a_{21}(L) \Delta \eta_{1 t}+a_{22}(L)\left(1-\left(1-\frac{c}{\sqrt{T}}\right) L\right) \eta_{2 t} \\
& =a_{21}(L) \Delta \eta_{1 t}+\tilde{a}_{22, T}(L) \eta_{2 t},
\end{aligned}
$$

where $\tilde{a}_{22, T}(L)=a_{22}(L)\left(1-\left(1-\frac{c}{\sqrt{T}}\right) L\right)$. By the Beveridge-Nelson decomposition, this can be rewritten as

$$
\Delta X_{2 t}=a_{21}(L) \Delta \eta_{1 t}+\tilde{a}_{22, T}(1) \eta_{2 t}+\tilde{a}_{22, T}^{*}(L) \Delta \eta_{2 t}
$$

with $\tilde{a}_{22, T}(1)=a_{22}(1) \frac{c}{\sqrt{T}}$ and $\tilde{a}_{22, T}^{*}(L)(1-L)=\tilde{a}_{22, T}(L)-\tilde{a}_{22, T}(1)$. Let us examine in more details this specification. The SMA bivariate representation contains a difference stationary process $\Delta X_{1 t}$ and a highly persistent process such that:

$$
\left[\begin{array}{l}
\Delta X_{1 t} \\
\Delta X_{2 t}
\end{array}\right]=\left[\begin{array}{cc}
a_{11}(L) & a_{12}(L)(1-L) \\
a_{21}(L)(1-L) & \tilde{a}_{22, T}(L)
\end{array}\right]\left[\begin{array}{l}
\eta_{1 t} \\
\eta_{2 t}
\end{array}\right]
$$


and only $\eta_{1 t}$ has a permanent effect on the first variable. The second variable $X_{2 t}$ is asymptotically stationary but locally nonstationary for values of $c$ greater than zero. ${ }^{10}$ The value of $c$ controls the amplitude of the local nonstationarity. Moreover, for appropriate values of $a_{21}(L)$ and $a_{22}(L)$, the process is asymptotically persistent but stationary. We also consider the case that the second shock can have locally a permanent effect on the first variable $X_{1 t}$. We show below that this can occur when the first variable is a linear function of the persistent variable $X_{2 t}$. For a fixed $T$, the corresponding characterization of the first variable is

$$
\begin{aligned}
\Delta X_{1 t} & =a_{11}(L) \eta_{1 t}+a_{12}(L)\left(1-\left(1-\frac{c}{\sqrt{T}}\right) L\right) \eta_{2 t} \\
& =a_{11}(L) \eta_{1 t}+\tilde{a}_{12, T}(L) \eta_{2 t}
\end{aligned}
$$

where $\tilde{a}_{12, T}(L)=a_{12}(L)\left(1-\left(1-\frac{c}{\sqrt{T}}\right) L\right)$ and $c$ is the same as in specification of the variable $X_{2 t}$. Here, the identification scheme based on the long run restriction that only the first shock has a permanent effect is still valid asymptotically but does not hold for a finite $T$. The resulting SMA bivariate representation is:

$$
\left[\begin{array}{l}
\Delta X_{1 t} \\
\Delta X_{2 t}
\end{array}\right]=\left[\begin{array}{cc}
a_{11}(L) & \tilde{a}_{12, T}(L) \\
a_{21}(L)(1-L) & \tilde{a}_{22, T}(L)
\end{array}\right]\left[\begin{array}{c}
\eta_{1 t} \\
\eta_{2 t}
\end{array}\right]
$$

An illustrative RBC model presented below motivates the proposed SMA representations (3) and (4).

\subsection{An illustrative RBC model}

The SMA representations (3) and (4) nest several business cycle models wherein TFP contains an exact unit root. To see this, let us consider the following simple dynamic model. The intertemporal expected utility function of the representative household is given by

$$
E_{t} \sum_{i=0}^{\infty} \beta^{i}\left\{\log \left(C_{t+i}\right)+B\left(1-H_{t+i}\right)\right\},
$$

where $B$ is a positive scale parameter, $\beta \in(0,1)$ denotes the discount factor and $E_{t}$ is the expectation operator conditional on the information set available as of time $t . C_{t}$ is the consumption at $t$ and $H_{t}$ represents the household's labor supply. Time endowment is normalized to unity. To ease the computation of the solution, we assume that utility is linear in leisure (see Hansen, 1985). The representative firm uses capital $K_{t}$ and labor $H_{t}$ to produce the homogeneous final

\footnotetext{
${ }^{10}$ The case of $c$ smaller than zero are not allowed to exclude nonfundamentalness representations of $\Delta X_{2 t}$ in finite sample.
} 
good $Y_{t}$. The technology is represented by the following constant returns-to-scale Cobb-Douglas production function

$$
Y_{t}=K_{t}^{\theta}\left(Z_{t} H_{t}\right)^{1-\theta},
$$

where $\theta \in(0,1)$. The TFP, represented by $Z_{t}$, is assumed to follow an exogenous process of the form

$$
\log \left(Z_{t}\right)=\log \left(Z_{t-1}\right)+\left(\gamma_{z}-1\right)+\sigma_{z} \eta_{z t}
$$

where $\gamma_{z}>1$ is the gross growth rate of TFP, $\sigma_{z}>0$ and $\eta_{z t}$ is iid with zero mean and unit variance. The capital stock evolves according to the law of motion

$$
K_{t+1}=(1-\delta) K_{t}+I_{t}
$$

where $\delta \in(0,1)$ is the constant depreciation rate and $I_{t}$ denotes investment. Finally, the final good can be either consumed or invested

$$
Y_{t}=C_{t}+I_{t}
$$

The approximate solution of the model is obtained from a $\log$-linearization of the stationary equilibrium conditions around their deterministic steady state (see Appendix A for more details). This gives the following equation for hours:

$$
(1-\varphi L) \widehat{h}_{t}=\nu \sigma_{z} \eta_{z t}
$$

where a hat represents the relative deviation from steady-state. The parameter $\nu$ is positive and $\varphi \in(0,1)$ is the stable root of the log-linear version of the model. Hours worked increase after a technology improvement and go back steadily toward their steady-state. Notice that hours can display persistent fluctuations, but they are stationary since $\varphi<1$.

Suppose now that hours worked $\left\{h_{t}\right\}_{t=1}^{t=T}$ are observed by the econometrician for a given finite sample T. Observed hours evolves according to the following measurement equation

$$
h_{t}=\widehat{h}_{t}+h_{t}^{c}
$$

where $h_{t}$ is the observed realizations of hours worked in log and in deviation from its mean, $\widehat{h}_{t}$ is given by equation (5) (the solution of the business cycle model) and $h_{t}^{c}$ represents a low-frequency measurement error. This low-frequency measurement error is assumed to follow a near-stationary persistent process of the form

$$
\begin{aligned}
\phi(L) \Delta h_{t}^{c} & =\left(1-\left(1-\frac{c}{\sqrt{T}}\right) L\right) \sigma_{c} \eta_{c t} \\
& =\frac{c}{\sqrt{T}} \sigma_{c} \eta_{c t}+\left(1-\frac{c}{\sqrt{T}}\right) \sigma_{c} \Delta \eta_{c t}
\end{aligned}
$$


where $c>0, \sigma_{c}>0$ and and $\eta_{c t}$ is iid with zero mean and unit variance. In addition, $\eta_{c t}$ is assumed to be orthogonal to $\eta_{z t}$ (contemporaneously and for all leads and lags). The polynomial $\phi(L)$ has all its roots outside the unit circle. For $T$ finite $h_{t}^{c}$ contains a unit root. When $T$ goes to infinity for a given positive $c$, the process (7) reduces to

$$
\phi(L) h_{t}^{c}=\sigma_{c} \eta_{c t}
$$

So, in a finite sample, observed hours are nonstationary for $c>0$. This characterization of lowfrequency measurement error tries to capture the difficulty with an insufficient span of available data to consistently estimate the mean reverting property of the underlying process. The series seems nonstationary for the sample on hands while the underlying process is stationary as hours worked per capita. This specification supposes that the highly persistent component which is hardly approximated in finite sample depends on a shock orthogonal to the technology shock (ex: a labor wedge shock). This can also capture low-frequency movements in the standard measure of hours worked corresponding to sectoral shifts in hours and the changing age composition of the working-age population (Francis and Ramey, 2009). According to these authors, these lowfrequency comovements must be removed from the labor productivity growth and hours worked series.

Using the model's solution (5), the measurement equation (6) and the measurement error (7), hours in first difference admit the following moving-average representation

$$
\begin{aligned}
\Delta h_{t} & =\Delta \widehat{h}_{t}+\Delta h_{t}^{c} \\
& =\left(\frac{\nu}{1-\varphi L}\right) \sigma_{z} \Delta \eta_{z t}+\frac{c \phi(L)^{-1}}{\sqrt{T}} \sigma_{c} \eta_{c t}+\phi(L)^{-1}\left(1-\frac{c}{\sqrt{T}}\right) \sigma_{c} \Delta \eta_{c t}
\end{aligned}
$$

From (8), we obtain that the technology shock has no long-run effect on hours, whereas the low frequency shock on the measurement errors has a permanent effect on hours in finite sample.

Using this equation and other model's variables, we can now present some illustrative examples for SMA representations (3) and (4). Denoting $\eta_{1 t}=\eta_{z t}$ and $\eta_{2 t}=\eta_{c t}$, equation (8) can be rewritten in the form of the process for $X_{2 t}$, where

$$
\begin{aligned}
a_{21}(L) & =\left(\frac{\nu}{1-\varphi L}\right) \sigma_{z} \\
\tilde{a}_{22, T}(L) & =\frac{c \phi(L)^{-1}}{\sqrt{T}} \sigma_{c}+\phi(L)^{-1}\left(1-\frac{c}{\sqrt{T}}\right) \sigma_{c}(1-L)
\end{aligned}
$$

This type of representation for $X_{2 t}$ will be always maintained, i.e. hours worked represent the locally nonstationary variable under study. The variable $X_{1 t}$ can be interpreted in several ways, 
but examples that we consider maintain the central assumption that $X_{1 t}$ contains an exact unit root.

Example $1: \Delta X_{1 t}$ is the growth rate of TFP. In this case, the process for $X_{1 t}$ reduces to $a_{11}(L)=\sigma_{z}$ and $a_{12}(L)=0$. However, $\Delta X_{1 t}$ can be a contaminated measure of TFP, but the contamination has no long-run effect in finite sample. This case can be handle by imposing $a_{11}(L)=\sigma_{z}$ and $a_{12}(L)=a_{12}^{(0)}$, where $a_{12}^{(0)}$ is a non-zero scalar. A more general formulation for $a_{12}(L)$ can be also considered to account for long-lasting contamination effects.

Example $2: \Delta X_{1 t}$ is the output growth. Using the solution of the business cycle model (see Appendix A), the output growth is given by

$$
\Delta y_{t}=\sigma_{z} \eta_{z t}-\mu \frac{\sigma_{z} \Delta \eta_{z t}}{1-\varphi L}
$$

where $\mu$ is a constant parameter. This implies that $a_{11}(L)=\sigma_{z}-\mu \frac{\sigma_{z}(1-L)}{1-\varphi L}$ and $a_{12}(L)=0$. Notice that a very similar formulation can be obtained if we replace output growth by consumption or investment growth. Measurement errors can be easily included by assuming that $a_{12}(L)$ is nonzero. The central assumption that we maintain is that these measurement errors have no long-run effects on output in a finite sample. In a more complex model, $\Delta y_{t}$ can be affected by an other permanent shock than the technology shock (see Galí, 1999).

Example 3 An interesting case is when the nonstationary variable considered in the SVAR model is contaminated by the highly persistent variable. For example, the productivity variable used in most of SVARs with long-run restrictions is growth rate of the labor productivity (see Galí, 1999, Christiano, Eichenbaum and Vigfusson, 2004a, Francis and Ramey, 2005). To illustrate this, consider the case where the econometrician constructs a measure of productivity growth using the output growth given by (9) and the observed change in hours given by (8):

$$
\Delta y_{t}-\Delta h_{t}=\sigma_{z} \eta_{z t}-\left(\frac{\mu+\nu}{1-\varphi L}\right) \sigma_{z} \Delta \eta_{z t}-\frac{c \phi(L)^{-1}}{\sqrt{T}} \sigma_{c} \eta_{c t}-\phi(L)^{-1}\left(1-\frac{c}{\sqrt{T}}\right) \sigma_{c} \Delta \eta_{c t}
$$

In this case, $\Delta X_{1 t}=\Delta y_{t}-\Delta h_{t}$ with $\Delta X_{2 t}=\Delta h_{t}$. Consequently, labor productivity is contaminated by the low-frequency component of hours. In other words, the variable $X_{1 t}$ is now function of the nearly stationary persistent process $X_{2 t}$. In finite sample, the two shocks $\eta_{z t}$ and $\eta_{c t}$ affect permanently the labor productivity. Francis and Ramey (2009) have already investigated this issue by showing that long-run identifying assumption used in empirical works are valid only when labor 
productivity are defined in efficiency unit. When they are not, labor productivity depends both on TFP and the ratio of efficiency hours to aggregate hours. In our statistical setup, the effect of this ratio in finite sample is captured by the near stationarity in the measurement errors. It is important to note here that output growth does not share the low-frequency movements of hours precluding a cointegration relationship.

\section{Estimation and Inference}

We now consider estimation and inference with the two specifications of the SVAR model. In practice, the reduced-form moving average representation is retrieved by performing a finite order VAR on the data. Suppose now that the structural moving average representation can be characterized or approximated in small sample by a finite VAR of order $p .{ }^{11}$ Consider, the following reduced form $\operatorname{VAR}(\mathrm{p})$ :

$$
D(L) X_{t}=\varepsilon_{t} \quad \text { where } \quad D(L)=I-\sum_{i=1}^{p} D_{i} L^{i}=\left[\begin{array}{cc}
1-\sum_{i=1}^{p} d_{11}^{(i)} L^{i} & -\sum_{i=1}^{p} d_{12}^{(i)} L^{i} \\
-\sum_{i=1}^{p} d_{21}^{(i)} L^{i} & 1-\sum_{i=1}^{p} d_{22}^{(i)} L^{i}
\end{array}\right] .
$$

By multiplying both sides by a matrix $B_{0}=\left[\begin{array}{cc}1 & -b_{12}^{(0)} \\ -b_{21}^{(0)} & 1\end{array}\right]=A_{0}^{-1}$, we obtain the VAR in function of the structural shocks as follows: $B(L) X_{t}=\eta_{t}$ with $B(L)=B_{0} D(L)$. More explicitly, the first variable is given by:

$$
\Delta X_{1 t}=\left(\sum_{i=1}^{p} d_{11}^{(i)} L^{i}-b_{12}^{(0)} \sum_{i=1}^{p} d_{21}^{(i)} L^{i}\right) \Delta X_{1 t}+\left(\sum_{i=1}^{p} d_{12}^{(i)} L^{i}+b_{12}^{(0)}\left[1-\sum_{i=1}^{p} d_{22}^{(i)} L^{i}\right]\right) X_{2 t}+\eta_{1 t} .
$$

Imposing the structural long-run impact matrix $A(1)$ to be lower triangular implies that $B_{0} D(1)$ is also lower triangular by $A(1)=D(1)^{-1} A_{0}$. The long-run multiplier of the variable $X_{2 t}$ on $\Delta X_{1 t}$ is then zero. Imposing this constraint yields

$$
\begin{aligned}
\Delta X_{1 t} & =\left(\sum_{i=1}^{p} d_{11}^{(i)} L^{i}-b_{12}^{(0)} \sum_{i=1}^{p} d_{21}^{(i)} L^{i}\right) \Delta X_{1 t}+b_{12}^{(0)} \Delta X_{2 t}+\sum_{i=1}^{p-1} \widetilde{b}_{12}^{(i)} L^{i} \Delta X_{2 t}+\eta_{1 t} \\
& =b_{11}(L) \Delta X_{1 t-1}+b_{12}^{(0)} \Delta X_{2 t}+\widetilde{b}_{12}(L) \Delta X_{2 t-1}+\eta_{1 t}
\end{aligned}
$$

with $b_{12}^{(0)}=-d_{12}(1) /\left(1-d_{22}(1)\right)$ and $\widetilde{b}_{12}^{(i)}=-\sum_{j=i+1}^{p}\left(d_{12}^{(j)}-b_{12}^{(0)} d_{22}^{(j)}\right)$. The second equation is:

$$
X_{2 t}=\left(b_{21}^{(0)}\left[1-\sum_{i=1}^{p} d_{11}^{(i)} L^{i}\right]+\sum_{i=1}^{p} d_{12}^{(i)} L^{i}\right) \Delta X_{1 t}+\left(\sum_{i=1}^{p} d_{22}^{(i)} L^{i}-b_{21}^{(0)} \sum_{i=1}^{p} d_{12}^{(i)} L^{i}\right) X_{2 t}+\eta_{2 t}
$$

\footnotetext{
${ }^{11}$ By considering the order $p \rightarrow \infty$ (at some rate) and $\frac{p}{T} \rightarrow 0$, Lewis and Reinsel (1985) show that a multivariate infinite autoregression can be arbitrarily approximated by a finite VAR of order $p$.
} 
which can be rewritten as

$$
\begin{aligned}
X_{2 t} & =\left(b_{21}^{(0)}\left[1-\sum_{i=1}^{p} d_{11}^{(i)} L^{i}\right]+\sum_{i=1}^{p} d_{12}^{(i)} L^{i}\right) \Delta X_{1 t}+b_{22}(1) X_{2 t-1}+\sum_{i=1}^{p-1} \widetilde{b}_{22}^{(i)} L^{i} \Delta X_{2 t}+\eta_{2 t} \\
& =b_{21}^{0} \Delta X_{1 t}+b_{21}(L) \Delta X_{1 t-1}+b_{22}(1) X_{2 t-1}+\widetilde{b}_{22}(L) \Delta X_{2 t-1}+\eta_{2 t}
\end{aligned}
$$

where $b_{22}(1)=d_{22}(1)-b_{21}^{(0)} d_{12}(1)$ and $\widetilde{b}_{22}^{(i)}=-\sum_{j=i+1}^{p} b_{22}^{(j)}=-\sum_{j=i+1}^{p}\left(d_{22}^{(j)}-b_{12}^{(0)} d_{12}^{(j)}\right)$.

\subsection{The LSVAR Model}

Let us first consider the LSVAR specification. With this specification, the second variable $X_{2 t}$ is included in the VAR in level. To simplify suppose that the initial condition is fixed to zero (i.e. $\left.X_{20}=0\right)$, the structural highly persistent process is rewritten in level as:

$$
X_{2 t}=a_{21}(L) \eta_{1 t}+a_{22}(1) \frac{c}{\sqrt{T}} \sum_{i=1}^{t} \eta_{2 i}+\tilde{a}_{22, T}^{*}(L) \eta_{2 t} .
$$

For a finite $T$ and $c>0$, the second structural shock has a permanent effect on the variable $X_{2 t}$. The finite measure of the element $(2,2)$ of the matrix $A(1)$ is then very sensitive to the value of the parameter $c$. For $T$ going to infinity and $c>0$, the second term at the RHS of eq. (11) does not disappear asymptotically but converges toward a Brownian motion. Thus,

$$
a_{22}(1) \frac{c}{\sqrt{T}} \sum_{i=1}^{[T r]} \eta_{2 i} \stackrel{L}{\longrightarrow} a_{22}(1) c W(r)
$$

where $W(r)$ is a standard Brownian motion for $r \in[0,1], t=[\mathrm{Tr}]$ and [.] means the integer part. Consequently, even though $\frac{1}{\sqrt{T}} \rightarrow 0$ as $T$ is going to infinity, the variance of $X_{2 t}$ is still function on the second term and in particular depends on the value $c$. We will show below that this introduces an asymptotic non standard distribution for impulse responses resulting from the LSVAR. For the first variable $\Delta X_{1 t}$, when the specification (3) is true, the long-run restriction that only the first structural shock has a permanent effect on the first variable is valid for all finite $T$. When the first variable is contaminated by the highly persistent process $X_{2 t}$ as labor productivity, the restrictions is violated for a finite $T$ as in specification (4) which introduces a potential finite sample bias of the long-run matrix $A(1)$ estimator. This finite sample bias is transmitted to the estimator of $A_{0}$ and the resulting impulse responses by the following relationships: $A(0)=C(1)^{-1} A(1)$ and $A(L)=C(L) A(0)$. This explains why dynamic responses from LSVAR using a "purified" measure of TFP differ from LSVAR using labor productivity and why the latter yields biased dynamic responses as shown in simulations by Chari, Kehoe and Mcgrattan (2008) and Fève and Guay (2010). 
Let us now examine more precisely the asymptotic properties of the LSVAR. We are interested by the estimation of the two following equations.

$$
\begin{aligned}
\Delta X_{1 t} & =b_{11}(L) \Delta X_{1 t-1}+b_{12}^{(0)} \Delta X_{2 t}+\widetilde{b}_{12}(L) \Delta X_{2 t-1}+\eta_{1 t} \\
X_{2 t} & =b_{21}^{0} \Delta X_{1 t}+b_{21}(L) \Delta X_{1 t-1}+b_{22}(1) X_{2 t-1}+\widetilde{b}_{22}(L) \Delta X_{2 t-1}+\eta_{2 t} .
\end{aligned}
$$

We rewrite the model as function of $b_{12}^{(0)}$ and $b_{21}^{(0)}$ whose asymptotic properties determines the limiting behavior of the impulse responses at the impact and $b_{22}^{*}$ defined as $b_{22}(1)-1$ above which undermines the asymptotic properties of the dynamic of impulse responses. Thus, the model can be rewritten up to $o_{p}(1)$ terms as

$$
\begin{aligned}
\Delta \widetilde{X}_{1 t} & =b_{12}^{(0)} \Delta \widetilde{X}_{2 t}+\eta_{1 t} \\
\Delta \widetilde{X}_{2 t} & =b_{21}^{(0)} \Delta \widetilde{X}_{1 t}+b_{22}^{*} \widetilde{X}_{2 t-1}+\eta_{2 t}
\end{aligned}
$$

where $\Delta \widetilde{X}_{1 t}, \Delta \widetilde{X}_{2 t}$ and $\widetilde{X}_{2 t-1}$ are defined as the residuals of the projection of these variables on the predetermined variables $W_{t-1}=\left(\Delta X_{1 t-1}, \ldots, \Delta X_{1 t-p}, \Delta X_{2 t-1}, \ldots, \Delta X_{2 t-p-1}\right)^{\prime}$ and $b_{22}^{*}=$ $b_{22}(1)-1$. To study the properties of the impulse responses, we consider the IV estimator of the SVAR model proposed by Shapiro and Watson (1988). Christiano, Eichenbaum and Vigfusson (2006) and Gospodinov (2010) employed this estimator to analyze the cases where the second variable is difference stationary or a nearly nonstationary process, respectively. The IV estimator of $b_{12}^{(0)}$ with $X_{2 t-1}$ as instrument is given by the following expression:

$$
\widehat{b}_{12}^{(0)}=\frac{\frac{1}{T} \sum_{t=2}^{T} X_{2 t-1} \Delta \widetilde{X}_{1 t}}{\frac{1}{T} \sum_{t=2}^{T} X_{2 t-1} \Delta \widetilde{X}_{2 t}}=\frac{\frac{1}{T} \sum_{t=2}^{T} X_{2 t-1}\left[b_{12}^{(0)} \Delta \widetilde{X}_{2 t}+\eta_{1 t}\right]}{\frac{1}{T} \sum_{t=2}^{T} X_{2 t-1} \Delta \widetilde{X}_{2 t}}
$$

or equivalently

$$
\widehat{b}_{12}^{(0)}-b_{12}^{(0)}=\frac{\frac{1}{T} \sum_{t=1}^{T} X_{2 t-1} \eta_{1 t}}{\frac{1}{T} \sum_{t=1}^{T} X_{2 t-1} \Delta \widetilde{X}_{2 t}}+o_{p}(1) .
$$

Consider now the estimation of the structural parameter $b_{21}^{(0)}$ in equation (15). Since $\eta_{1 t}$ and $\eta_{2 t}$ are orthogonals, the residuals $\widehat{\eta}_{1 t}=\Delta \widetilde{X}_{1 t}-\widehat{b}_{12}^{(0)} \Delta \widetilde{X}_{2 t}$ can be used as instrument for the endogenous variable $\Delta \widetilde{X}_{1 t}$. Thus, $\widehat{\eta}_{1 t}=\eta_{1 t}-\left(\widehat{b}_{12}^{(0)}-b_{12}^{(0)}\right) \Delta \widetilde{X}_{2 t}$. Let us now define $z_{t}=\left(\widehat{\eta}_{1 t}, X_{2 t-1}\right)^{\prime}$ and $x_{t}=$ $\left(\Delta \widetilde{X}_{1 t}, \widetilde{X}_{2 t-1}\right)^{\prime}$. The IV estimator of $\beta=\left(b_{21}^{(0)}, b_{22}^{*}\right)^{\prime}$ is then given by $\widehat{\beta}=\left[\frac{1}{T} \sum_{t=2}^{T} z_{t} x_{t}^{\prime}\right]^{-1}\left[\frac{1}{T} \sum_{t=2}^{T} z_{t} \Delta \widetilde{X}_{2 t}\right]$. The following theorem provides the asymptotic properties for the IV estimator of $b_{12}^{(0)}, b_{21}^{(0)}$ and $b_{22}^{*}$. 
Theorem 1 Under the structural model (3) or (4) and assumptions in Lemma 1, the IV estimator $\widehat{b}_{12}^{(0)} \stackrel{p}{\rightarrow} b_{12}^{(0)}$ and $\widehat{\beta} \stackrel{p}{\rightarrow} \beta$ converges to the true value. Thus,

$$
\begin{gathered}
\sqrt{T}\left(\widehat{b}_{12}^{(0)}-b_{12}^{(0)}\right) \stackrel{L}{\longrightarrow} \frac{\left(\psi_{2,0}^{1 / 2} \xi_{1}+\vartheta_{1}\right)}{a_{22}^{(0)} b_{22}^{*} \widetilde{\psi}_{2}}, \\
\sqrt{T}\left(\widehat{b}_{21}^{(0)}-b_{21}^{(0)}\right) \stackrel{L}{\longrightarrow} \frac{\xi_{2}}{a_{11}^{(0)}}-\frac{\left(\psi_{2,0}^{1 / 2} \xi_{1}+\vartheta_{1}\right)}{a_{22}^{(0)} b_{22}^{*} \widetilde{\psi}_{2}} . \\
\sqrt{T}\left(\widehat{b}_{22}^{*}-b_{22}^{*}\right) \stackrel{L}{\longrightarrow} \frac{a_{12}^{(0)}\left(\psi_{2,0}^{1 / 2} \xi_{1}+\vartheta_{1}\right)}{a_{11}^{(0)} \widetilde{\psi}_{2}}-\left[\frac{a_{12}^{(0)} b_{22}^{*}}{a_{11}^{(0)}}-\frac{\psi_{2,0}^{1 / 2}}{\widetilde{\psi}_{2}}\right] \xi_{2}+\frac{\vartheta_{2}}{\widetilde{\psi}_{2}}
\end{gathered}
$$

where $\vartheta_{1}=a_{22}(1) c \int_{0}^{1} W_{2}(r) d W_{1}(r), \vartheta_{2}=a_{22}(1) c \int_{0}^{1} W_{2}(r) d W_{2}(r)$ with $\xi=\left(\xi_{1}, \xi_{2}\right)^{\prime} \sim \mathcal{N}\left(0, I_{2}\right)$, $W_{1}(r)$ and $W_{2}(r)$ are two independent Brownian motions and $\widetilde{\psi}_{2}$ and $\psi_{2,0}^{1 / 2}$ are defined in the Appendix.

Theorem 1 establishes that the IV estimator of $b_{12}^{(0)}, b_{21}^{(0)}$ and $b_{22}^{*}$ are consistent. This result is not surprising since as $T$ goes to infinity, $X_{2 t}$ is a second order stationary variable. The second set of result shows that the asymptotic distribution of $\sqrt{T}\left(\widehat{b}_{12}^{(0)}-b_{12}^{(0)}\right)$ is a mixture of Gaussian distributions for $c$ greater than zero. The first term of the asymptotic distribution is the usual asymptotic distribution for a stationary variable $X_{2 t}$. The term $\vartheta_{1}$ has a Gaussian distribution conditional on $W_{2}(\cdot)$ since $W_{1}(\cdot)$ is independent of $W_{2}(\cdot)$. This component produces wider confidence interval compared to the standard case with stationary variables by increasing the parameter $c$ controling the local nonstationarity. Theorem 1 also shows that the asymptotic distribution of the IV estimator of $b_{21}^{(0)}$ is a function of the asymptotic distribution of $b_{12}^{(0)}$ and then shares the same asymptotic properties. This is due to the use of the instrument which depends on the estimator $\widehat{b}_{12}^{(0)}$. Finally, the asymptotic distribution of the IV estimator of persistence parameter $b_{22}^{*}$ is non standard and depends on $\vartheta_{1}$ and $\vartheta_{2}$. The term $\vartheta_{2}$ is of an unit-root type distribution providing fat tails in the asymptotic distribution. Larger is the value of $c$, wider is the non standard confidence interval.

In the light of the business cycle model, Theorem 1 states that whatever the variable used for $X_{1 t}$ (TFP, output or a proper measure of labor productivity), the LSVAR provides consistent estimators. The definition of $X_{1 t}$ does not matter a lot for the asymptotic distribution. To see this, let us consider the case where $\Delta X_{1 t}$ is the growth rate of TFP, i.e. $\Delta X_{1, t}=\Delta z_{t}$. This implies that $a_{12}^{(0)}=0$ and thus $b_{12}^{(0)}=0$. In this case, the limiting distribution of $\widehat{b}_{12}^{(0)}$ and $\widehat{b}_{21}^{(0)}$ are left unaffected. 
The sole difference concerns $\widehat{b}_{22}^{*}$ for which the limiting distribution does not include the random variables $\zeta_{1}$ and $v_{1}$. However, the asymptotic distribution is still non standard as it depends on $v_{2}$. Again, larger values of $c$ tends to increase the confidence interval for $\hat{b}_{22}^{*}$.

We now examine the asymptotic behavior of the impulse response function to the permanent shock (e.g, $\left.\eta_{1 t}\right)$. According to the SMA representation (2), let $a_{k j}^{(l)}=\frac{\partial X_{k, t+l}}{\partial \eta_{j t}}$ be the impulse response function at $l$ periods ahead for a normalized structural shock $j$ for the variable $X_{k}$. Since the estimators of $b_{12}^{(0)}, b_{21}^{(0)}$ and $b_{22}^{*}$ are consistent according to Theorem 1 and other parameters in the VAR are also consistently estimated, we can show that estimates of impulse responses $a_{k j}^{(l)}$ resulting from the VAR are also consistent. However, their asymptotic distributions are non standard due to the local nonstationarity of $X_{2 t}$. A simple example can give insight about the effect of the local nonstationary on the asymptotic distribution of the impulse responses. Consider the following $\operatorname{VAR}(1)$ model

$$
\begin{aligned}
\Delta X_{1 t} & =b_{12}^{(0)} \Delta X_{2 t}+\eta_{1 t} \\
X_{2 t} & =b_{21}^{(0)} \Delta X_{1 t}+b_{22} X_{2 t-1}+\eta_{2 t} .
\end{aligned}
$$

For instance, in this case the estimator of the impulse response function of the first shock on the second variable $X_{2 t}$, this is given by

$$
\widehat{a}_{21}^{(l)}=\frac{\widehat{b}_{21}^{(0)}\left(\widehat{b}_{22}-\widehat{b}_{12}^{(0)} \widehat{b}_{21}^{(0)}\right)^{l}}{\left(1-\widehat{b}_{12}^{(0)} \widehat{b}_{21}^{(0)}\right)^{l+1}} .
$$

and $\widehat{b}_{22}^{*}=\widehat{b}_{22}-1$. At the impact (i.e. $l=0$ ), the asymptotic distribution depends only on the asymptotic distribution of the parameters $b_{12}^{(0)}$ and $b_{21}^{(0)}$ which is a scaled mixed of Gaussian distributions depending on $c$. For the propagation of the shocks (i.e. $l>0$ ), the asymptotic distribution depend also on the asymptotic distribution of $b_{22}^{*}$ which is of the unit-root distribution inducing fat-tails distribution for the impulse responses. In the more specific case where $\Delta X_{1 t}$ is the growth rate of the TFP, $b_{12}^{(0)}=0$, the estimator of the impulse response function of the first shock on the second variable $X_{2 t}$ is given by the following simple expression

$$
\widehat{a}_{21}^{(l)}=\widehat{b}_{21}^{(0)}\left(\widehat{b}_{22}^{*}-1\right)^{l} \text {. }
$$

\subsection{The DSVAR Model}

Let us now consider the DSVAR specification. From (4), the finite sample measure of the long-run impact is given by:

$$
A_{T}(1)=\left[\begin{array}{cc}
a_{11}(1) & a_{12}(1) c / \sqrt{T} \\
0 & a_{22}(1) c / \sqrt{T}
\end{array}\right]
$$


When the first variable $\Delta X_{1 t}$ is not function of the second variable as in (3), the term $a_{12}(1) c / \sqrt{T}$ is equal to zero and the long-run restriction that only the first shock has a permanent effect on the first variable is valid. When, the first variable corresponds to labor productivity growth, since $\Delta X_{1 t}=\Delta y_{t}-\Delta h_{t}$ and $\Delta X_{2 t}=\Delta h_{t}$ implies $a_{12}(1) c / \sqrt{T}=-a_{22}(1) c / \sqrt{T}$. The linear dependence between the labor productivity growth and $\Delta h_{t}$ induces negative low frequency co-movements between these variables. In contrast to small low-frequency co-movements discuss in Gospodinov, Maynard and Pesavento (2011) which are present in the LSVAR specification but disappear in the DSVAR specification, the negative co-movements here are present in the DSVAR specification. ${ }^{12}$ In particular, the negative low-frequency co-movements between labor productivity and hours in the DSVAR which are absent in a DSVAR including TFP and hours explain the difference in the estimated impulse responses between these two specifications.

As $T \rightarrow \infty$, the long-run restriction that only the first shock has a permanent effect on the first variable is valid. However, this long-run restriction measured by the matrix $A(1)$ is violated for a finite $T$ and this matrix is upper triangular instead of lower triangular. Provided $c>0$ and $T$ fixed, two shocks have a long-run effect on $X_{1 t}$, the permanent shock $\eta_{1 t}$ and the non-permanent $\eta_{2 t}$. Suppose now that we use, as usual, a long-run identification scheme to uncover $\eta_{1 t}$. It follows that the long-run effect of $\eta_{2 t}$ on $X_{1 t}$ will be attributed to $\eta_{1 t}$, leading to over-estimate the contribution of $\eta_{1 t}$. Notice that when $c$ increases, i.e. when the variable $X_{2 t}$ becomes more and more persistent, the effect of $\eta_{2 t}$ on $X_{1 t}$ increases, because the variable $X_{1 t}$ is contaminated by $X_{2 t}$. Indeed, the second shock $\eta_{2 t}$ will have a permanent effect on $X_{2 t}$ in finite sample. When the size of the local nonstationary alternative $c$ increases, the shock $\eta_{2 t}$ will have larger permanent effect on $X_{2 t}$. Again, because the long-run identification will wrongly attribute to $\eta_{1 t}$ the permanent effect on $X_{1 t}$ of $\eta_{2 t}$, this identification scheme will conclude that the shock $\eta_{1 t}$ will have a permanent effect on $X_{2 t}$ whose sign will depend on the sign of $a_{12}(1)$. Observe also as $T \rightarrow \infty$, only the element $(1,1)$ is different from zero and the matrix $A_{\infty}(1)$ is singular resulting from the over-differentiation of $X_{2 t}$.

The next proposition characterizes more precisely the finite sample measure at zero frequency when a lower triangular structure is imposed to the matrix $A_{T}(1)$ for the DSVAR model.

\footnotetext{
${ }^{12}$ Using estimated DSVAR parameters, we can compute low-frequency co-movements between labor productivity growth and hours worked in difference using the non-farm business sector data for a given interval of frequencies and, in particular, for the frequency zero and its neighborhood. In fact, it exists a non negligible negative lowfrequency correlation between labor productivity growth and hours worked in difference. This is consistent with our specification.
} 
Proposition 1 Consider a DSVAR model with variables $\Delta X_{1 t}$ and $\Delta X_{2 t}$ defined in eq. (4). The finite sample measure of the long-run impact of the structural shocks by using the Choleski decomposition is given by the following lower triangular matrix :

$$
\operatorname{chol}\left(A_{T}(1) A_{T}(1)^{\prime}\right)=\left[\begin{array}{cc}
\left(a_{11}(1)^{2}+a_{12}(1)^{2} c^{2} / T\right)^{1 / 2} & 0 \\
\frac{a_{12}(1) a_{22}(1) c^{2} / T}{\left(a_{11}(1)^{2}+a_{12}(1)^{2} c^{2} / T\right)^{1 / 2}} & \left(a_{22}(1)^{2} c^{2} / T-\frac{a_{12}(1)^{2} a_{22}(1)^{2} c^{4} / T^{2}}{a_{11}(1)^{2}+a_{12}(1)^{2} c^{2} / T}\right)^{1 / 2}
\end{array}\right] .
$$

where $\operatorname{chol}(\cdot)$ is the Choleski decomposition.

Imposing that the second shock has no long-run effect on the variable $X_{1 t}$ yields a theoretical (population) impulse response function of the permanent shock $\eta_{1 t}$ that is biased. The finite sample bias introduced by wrongly imposing a lower triangular matrix depends on the parameter $a_{12}(1)$. First, the finite sample measure of the long-run impact of the permanent shock on the first variable is over-stated since $\left(a_{11}(1)^{2}+a_{12}(1)^{2} c^{2} / T\right)^{1 / 2}>a_{11}(1)$ for $a_{12}(1) \neq 0$. Second, the finite sample measure of the long-run impact of the first structural shock $\eta_{1 t}$ on the second variable is of the same sign as $a_{12}(1)$. For a negative value of $a_{12}(1)=-a_{22}(1)$, which corresponds to the case of labor productivity, the finite sample measure of the long run impact is negative. The contamination of the finite sample measure of the long-run impact is then transmitted to the estimator of the relationship matrix $A_{0}$ by the expression : $A_{0}=C(1)^{-1} A(1)$. This result is of importance since it establishes that the local nonstationarity of per capita hours worked may lead to a downward bias in the estimated dynamic responses from a DSVAR model (when $a_{12}(1)$ is negative), despite the fact that a first difference specification seems to be adequate in finite sample.

We can easily translate the results of the above Proposition in terms of the business cycle model. First, consider that $\Delta X_{1 t}$ is the growth of labor productivity given by (10) and the econometrician estimates a VAR model with a difference specification for observed hours $\Delta h_{t}$. From equations (10) and (8), the finite sample measure of the long-run impact matrix is given by:

$$
A_{T}(1)=\left[\begin{array}{cc}
\sigma_{z} & -\frac{c \phi(1)^{-1}}{\sqrt{T}} \sigma_{c} \\
0 & \frac{c \phi(1)^{-1}}{\sqrt{T}} \sigma_{c}
\end{array}\right]
$$

Now, applying a Choleski decomposition to the long-run covariance matrix $A_{T}(1) A_{T}(1)^{\prime}$, we obtain the long-run effect of each structural shocks as identified by the DSVAR model:

$$
\left.\operatorname{chol}\left(A_{T}(1) A_{T}(1)^{\prime}\right)=\left[\begin{array}{cc}
\left(\sigma_{z}^{2}+\frac{c^{2} \phi(1)^{-2} \sigma_{c}^{2}}{T}\right)^{1 / 2} & 0 \\
\left.-\frac{c^{2} \phi(1)^{-2} \sigma_{c}^{2}}{T\left(\sigma_{z}^{2}+\frac{c^{2} \phi(1)-2}{T} \sigma_{c}^{2}\right.}\right)^{1 / 2} & \left(\frac{c^{2} \phi(1)^{-2} \sigma_{c}^{2}}{T}-\frac{c^{4} \phi(1)^{-4} \sigma_{c}^{4}}{T^{2}\left(\sigma_{z}^{2}+\frac{c^{2} \phi(1)-2}{T} \sigma_{c}^{2}\right.}\right)
\end{array}\right)^{1 / 2}\right]
$$


First, looking at the $(1,1)$ entry of the decomposition, it appears that the long-run effect of identified technology shock on labor productivity is over-estimated in finite sample as long as $c>0$. Second, inspecting the $(2,1)$ entry, the DSVAR model will predict a negative long-run effect of technology shock on hours under a business cycle model in which this long-run effect is zero. When $T$ is large enough regarding $c$, the long-run effect tends to zero.

Another interesting results from Proposition 1 is the case where $a_{12}(L)=0$. It follows immediately that the long-run response of $X_{2 t}$ to $\eta_{1 t}$ is zero, whatever or not this variable follows a local non-stationary process. This can be illustrated again, using the business cycle model. Consider now that the econometrician uses a perfect measure of the TFP. ${ }^{13}$ In this case, $\Delta X_{1 t}=\Delta z_{t}$. From (8), the finite sample measure of the long-run impact matrix is now given by:

$$
A_{T}(1)=\left[\begin{array}{cc}
\sigma_{z} & 0 \\
0 & \frac{c \phi(1)^{-1}}{\sqrt{T}} \sigma_{c}
\end{array}\right]
$$

The Choleski decomposition of the long-run covariance matrix yields

$$
\operatorname{chol}\left(A_{T}(1) A_{T}(1)^{\prime}\right)=\left[\begin{array}{cc}
\sigma_{z} & 0 \\
0 & \frac{c \phi(1)^{-1}}{\sqrt{T}} \sigma_{c}
\end{array}\right] .
$$

The long-run responses of the TFP and hours obtained from the DSVAR model are consistent with the business cycle model, even in finite sample. The parameter $c$ does not affect the estimated long-run response of TFP and hours to a technology shock. This results is of importance because it corresponds to the case where $b_{12}^{(0)}=0$. As shown below, in this case, the short-run response obtained from a DSVAR are unbiased and the dynamic responses can display small bias if a sufficient number of lags are included in the VAR model. Coupled with estimated long-run response, our results indicate that DSVAR can almost properly uncover the dynamic responses of hours worked when the econometrician use a perfect measure of TFP.

To study in more details the properties of the estimators resulting for the DSVAR, the corresponding estimated reduced form $\operatorname{VAR}(\mathrm{p})$ for both variables in difference is given by:

$$
\mathbf{D}(\mathbf{L}) \mathbf{X}_{t}=\varepsilon_{t}
$$

where the vector $\mathbf{X}_{t}$ is now defined as $\mathbf{X}_{t}=\left(\Delta X_{1 t}, \Delta X_{2 t}\right)^{\prime}$. By multiplying both sides by $B_{0}=$ $\left[\begin{array}{cc}1 & -b_{12}^{(0)} \\ -b_{21}^{(0)} & 1\end{array}\right]=A_{0}^{-1}$, we obtain the VAR in function of the structural shocks: $\mathbf{B}(L) \mathbf{X}_{t}=\eta_{t}^{*}$ with $\mathbf{B}(L)=B_{0} \mathbf{D}(L)$. Imposing the structural long-run impact matrix to be lower triangular

\footnotetext{
${ }^{13}$ Similar results for long-run responses hold when measurement errors has no long-run effect on this measure in finite sample.
} 
implies that $B_{0} \mathbf{D}(1)$ is also lower triangular. The long-run multiplier of the variable $\Delta X_{2 t}$ on $\Delta X_{1 t}$ is then zero. Imposing this constraint yields for the first equation,

$$
\Delta X_{1 t}=\mathbf{b}_{11}(L) \Delta X_{1 t-1}+b_{12}^{(0)} \Delta^{2} X_{2 t}+\widetilde{\mathbf{b}}_{12}(L) \Delta^{2} X_{2 t-1}+\eta_{1 t}^{*}
$$

and for the second equation:

$$
\Delta X_{2 t}=b_{21}^{0} \Delta X_{1 t}+\mathbf{b}_{21}(L) \Delta X_{1 t-1}+\mathbf{b}_{22}(1) \Delta X_{2 t-1}+\widetilde{\mathbf{b}}_{22}(L) \Delta^{2} X_{2 t-1}+\eta_{2 t}^{*}
$$

Asymptotically, the LSVAR is correctly specified while the DSVAR is misspecified. To study the asymptotic properties of the estimators obtained with the DSVAR specification, we rewrite the correctly specified LSVAR's eq. (12) such that the variables $X_{2 t}$ and its lags appear in second difference as function of the parameter $b_{12}^{(0)}$. Thus

$$
\begin{aligned}
\Delta X_{1 t} & =b_{11}(L) \Delta X_{1 t-1}+b_{12}^{(0)} \Delta X_{2 t}+\widetilde{b}_{12}(L) \Delta X_{2 t-1}+\eta_{1 t} \\
& =b_{11}(L) \Delta X_{1 t-1}+b_{12}^{(0)} \Delta^{2} X_{2 t}+\widetilde{b}_{12}(L) \Delta^{2} X_{2 t-1}+\eta_{1 t}^{*}
\end{aligned}
$$

with $\eta_{1 t}^{*}=-b_{12}^{(0)} \Delta X_{2 t-1}-\widetilde{b}_{12}(L) \Delta X_{2 t-2}+\eta_{1 t}$. By comparing eq. (17) with eq. (19), we see that the error $\eta_{1 t}^{*}$ is function of the lagged values of $\Delta X_{2 t}$. By rewriting also the second equation of the LSVAR, we can compare with the second equation of the DSVAR. Thus,

$$
X_{2 t}=b_{21}^{0} \Delta X_{1 t}+b_{21}(L) \Delta X_{1 t-1}+b_{22}(1) X_{2 t-1}+\widetilde{b}_{22}(L) \Delta X_{2 t-1}+\eta_{2 t}
$$

and rewriting in difference

$$
\Delta X_{2 t}=b_{21}^{0} \Delta X_{1 t}+b_{21}(L) \Delta X_{1 t-1}+b_{22}(1) \Delta X_{2 t-1}+\widetilde{b}_{22}(L) \Delta^{2} X_{2 t-1}+\eta_{2 t}^{*}
$$

with $\eta_{2 t}^{*}=-b_{21}^{0} \Delta X_{1 t-1}-b_{21}(L) b_{22}(L) \Delta X_{1 t-2}+\eta_{2 t}-\eta_{2 t-1}$.

As we proceed for the LSVAR, the first equation of the DSVAR is rewritten as:

$$
\Delta \widetilde{X}_{1 t}=b_{12}^{(0)} \Delta^{2} \widetilde{X}_{2 t}+\eta_{1 t}^{*}
$$

where $\Delta \tilde{X}_{1 t}$ and $\Delta^{2} \widetilde{X}_{2 t}$ are defined as the residuals of the projection of these variables on the predetermined variables $\left(\Delta X_{1, t-1}, \ldots, \Delta X_{1, t-p}, \Delta^{2} X_{2 t-1}, \ldots, \Delta^{2} X_{2 t-p-1}\right)$. Using $\Delta X_{2 t-1}$ as instruments, the IV estimator of $b_{12}^{(0)}$ in equation (21) is then given by the following expression:

$$
\widehat{b}_{12}^{(0)}=\frac{\frac{1}{T} \sum_{t=2}^{T} \Delta X_{2 t-1} \Delta \widetilde{X}_{1 t}}{\frac{1}{T} \sum_{t=2}^{T} \Delta X_{2 t-1} \Delta^{2} \widetilde{X}_{2 t}} .
$$


Since $\Delta X_{2 t-1}$ and $\eta_{1 t}^{*}$ are correlated by eq. (19), the DSVAR estimator of $b_{12}^{(0)}$ is asymptotically biased. Consider now the estimation of the parameters of equation (18). As in the LSVAR case, one uses the residuals $\widehat{\eta}_{1 t}^{*}=\Delta \widetilde{X}_{1 t}-\widehat{b}_{12}^{(0)} \Delta^{2} \widetilde{X}_{2 t}$ as instrument for the endogenous variable $\Delta X_{1 t}$.

Thus, $\widehat{\eta}_{1 t}^{*}=\eta_{1 t}^{*}-\left(\widehat{b}_{12}^{(0)}-b_{12}^{(0)}\right) \Delta^{2} \widetilde{X}_{2 t}$. The estimator is obviously asymptotically biased by the correlation between $\eta_{1 t}^{*}$ and $\eta_{2 t}^{*}$ as we can see by expressions derived above. The estimator of $b_{22}(1)$ is also not consistent by the correlation between $\Delta X_{2 t-1}$ and $\eta_{2 t}^{*}$.

Now, suppose that the DSVAR is estimated with a "purified" of TFP. In this case, the first equation of the DSVAR is given by:

$$
\Delta X_{1 t}=b_{11}(L) \Delta X_{1 t-1}+\eta_{1 t}^{*}
$$

The first equation of the DSVAR is now the same as the LSVAR. The IV estimator $\widehat{b}_{12}^{(0)}$ defined above is then consistent and converge to zero since $\eta_{1 t}^{*}=\eta_{1 t}$ in this case. The resulting residuals $\widehat{\eta}_{1 t}^{*}$ can be used as instruments in the second equation for the estimation of the parameter $b_{21}^{(0)}$. This also yields a consistent estimator of $b_{21}^{(0)}$ thanks to the absence of correlation between $\widehat{\eta}_{1 t}^{*}$ and $\eta_{2 t}^{*}$. However the estimator of $b_{22}(1)$ is still inconsistent. The asymptotic bias of the estimator of $b_{22}(1)$ is function of the following term: $\lim _{T \rightarrow \infty} \frac{1}{T} \Delta X_{2 t-1} \eta_{2 t}^{*}$. An important part of this bias comes from the unit root in the error term $\Delta \eta_{2 t}$ resulting from the overdifferentiation of the second equation of the VAR. This bias can be reduced by increasing the number of lags in the DSVAR model (see Marcet, 2005).

\section{Simulation Experiments from a DSGE Model with Real Fric- tions}

This section provides simulation experiments from an DSGE model calibrated and estimated with US data. ${ }^{14}$ The model used for the simulations extends to one introduced in Section 2.2 by allowing for habits in consumption and investment adjustment costs. Both mechanisms have proven useful in accounting for the dynamics of macroeconomic variables in particular in terms of their persistence properties (see e.g. Beaudry and Guay, 1996, Boldrin, Christiano and Fisher, 2001 and Christiano, Eichenbaum and Evans, 2005). Intertemporal consumption choices are not time separable and the flows of consumption services are a linear function of current and lagged consumption decisions.

\footnotetext{
${ }^{14}$ See Erceg, Guerrieri and Gust (2005), Chari, Kehoe and Mc Grattan (2008), Christiano, Eichenbaum and Vigfusson (2006) and Fève and Guay $(2009,2010)$ for other simulation experiments.
} 
The intertemporal expected utility function of the representative household is now given by

$$
E_{t} \sum_{i=0}^{\infty} \beta^{i}\left\{\log \left(C_{t+i}-b C_{t+i-1}\right)-\frac{H_{t+i}^{1+\nu}}{1+\nu}\right\}
$$

where $\nu$ is the inverse of the Frisch labor supply elasticity. Concerning the technology side, we adopt exactly the same specification as the one adopted in Section 2.2. Remind that TFP is assumed to follow a random walk process with drift. The homogenous produced good $Y_{t}$ can be used for consumption $C_{t}$ and investment $I_{t}$ purposes. Capital accumulation is governed by the following law of motion

$$
K_{t+1}=(1-\delta) K_{t}+\left[1-S\left(\frac{I_{t}}{I_{t-1}}\right)\right] v_{t} I_{t}
$$

where $\delta \in(0,1)$ is the constant depreciation rate and $S($.$) reflects the presence of adjustment costs.$ We assume that $S\left(\right.$.) satisfies (i) $S\left(\gamma_{z}\right)=S^{\prime}\left(\gamma_{z}\right)=0$ and (ii) $\xi=S^{\prime \prime}\left(\gamma_{z}\right) \gamma_{z}^{2}>0$. It follows that the steady state of the model does not depend on the parameter $\xi$ while its dynamic properties do. As in Smets and Wouters (2007), the variable $v_{t}$ represents a disturbance to the investment-specific technology process and is assumed to follow a first order autoregressive process

$$
\log \left(v_{t}\right)=\rho_{v} \log \left(v_{t-1}\right)+\sigma_{b} \eta_{v, t}
$$

where $\left|\rho_{v}\right|<1, \sigma_{v}>0$ and $\eta_{v, t}$ is iid with zero mean and unit variance.

As usual, the model is deflated for the stochastic trend component $Z_{t}$ and log-linearized around the deterministic steady state to obtain a state-space representation. Let $\Psi$ denotes the whole set of model parameters. The parameters of the state-space solution of the model depends on complicated nonlinear functions of $\Psi$. We split $\Psi$ in two vectors $\Psi_{1}$ and $\Psi_{2}$. The first vector $\Psi_{1}=\{\beta, \alpha, \delta, \nu\}$ includes parameters which are calibrated for the US economy prior to estimation. The discount factor $\beta$ is chosen such that the steady-state annual return to capital equals $3.6 \%$. The elasticity of output to the labor input $1-\alpha$ equals 0.67 , which corresponds to the average share of labor income to output. The depreciation rate of physical capital $\delta$ is set equal to 0.0153 . The value of $\nu=2$ in the utility function is set according to previous estimates with US data (see Smets and Wouters, 2007). All theses values are reported in the first column of Table 2. The second vector $\Psi_{2}=\left\{b, \xi, \gamma_{z}, \sigma_{z}, \rho_{v}, \sigma_{v}, \rho_{c}, c, \sigma_{c}\right\}$ contains the parameters which summarize the real frictions of the model (habits in consumption $b$ and the dynamic adjustment cost $\xi$ ), the law of motion of the two structural shocks $\left(\gamma_{z}, \sigma_{z}, \rho_{v}\right.$ and $\left.\sigma_{v}\right)$ and the measurement error. As in our illustrative model, we assume that actual hours differ from those of the model by a measurement error $h_{t}^{c}$ that follow 
the process

$$
\left(1-\rho_{c} L\right) \Delta h_{t}^{c}=\left(1-\left(1-\frac{c}{\sqrt{T}}\right) L\right) \sigma_{c} \eta_{c t}
$$

where $\left|\rho_{c}\right|<1, \sigma_{c}>0$ and $\eta_{c t}$ is iid with zero mean and unit variance. When $c>0$, this measurement error is non stationary in small sample (when $T$ is fixed), but asymptotically stationary (when $T \rightarrow \infty$ ).

From the state-space representation resulting from the log-linearized version of the model and under the assumption of Gaussian shocks, the log-likelihood function can be evaluated. The parameters of vector $\Psi_{2}$ are then estimated by maximizing this function. We use US quarterly data covering the sample period 1948Q1-2007Q4. The observed variables are the growth rate of the real GDP, real consumption expenditures (non-durable \& services) and total hours worked (per capita). Total hours worked are borrowed from Francis and Ramey (2009). The estimation results are reported in the second column of Table 2. The parameters are precisely estimated and are in the line of previous estimations for the US economy (see Smets and Wouters, 2007). The habit persistence parameter $b$ is positive and significant. The adjustment cost parameter $\xi$ takes a large value. These estimated values are crucial in replicating US data, especially the serial correlation of output growth and the log of hours. For example, setting $b=\varphi=0$ dramatically reduces the log-likelihood and a likelihood ratio test strongly rejects this restriction. In other words, our estimation results favor a version of the model with a sizeable amount of real frictions. The investment shock exhibits small persistence but its standard error is significantly higher than the one for the permanent technology shock. Finally, the estimated parameters of the measurement error rejects a pure random-walk representation.

Using estimated values, we compute the dynamic responses of hours worked following a technology shock implied by the model (see Figure 4). Hours worked decrease on impact and its response turns out to be persistently positive after one year. These findings are again in accordance with those obtained from estimated DSGE models (see Smets and Wouters, 2007), from SVAR models (see Gali, 1999, Francis and Ramey, 2008) and direct measures of TFP (see Basu, Fernald and Kimball, 2006). In our model, this response of hours is the result of the interplay between habit persistence in consumption and adjustment costs on investment. As pointed out by Francis and Ramey (2005), strong enough habit persistence induces a sluggish response of consumption. Facing a positive technology shock, households can put the extra resources on investment. However, the high degree of adjustment cost on capital implies that an additional investment is very costly. Consequently, households choose to spend their new wealth on the only remaining choice, i.e. they 
increase their leisure. We also use the estimated DSGE model in order to compute some statistics which summarize the time series behavior of two measures of hours worked: the first, labeled contaminated hours, includes the measurement errors and the second, labeled uncontaminated hours, is directly obtained from the estimated DSGE model. Consequently, we obtain two measures of labor productivity depending on the hours worked measure. We evaluate the contribution of the technology shock to labor productivity growth and change in hours worked. This shock accounts for a small portion of fluctuations in contaminated hours worked since it represents $12.43 \%$ of their variance. Interestingly, the technology shock explains $51.19 \%$ of the labor productivity growth. This is in contrast with the uncontaminated measure of hours. In this case, the technology shocks accounts for $73.04 \%$ of labor productivity growth. The computation of the autocorrelation function of both measures of hours are reported in Figure 5. For comparison purpose this figure includes the autocorrelation function of total and adjusted hours of Francis and Ramey (2009). This figure clearly shows that the contaminated measure of hours displays more persistence than the uncontaminated measure, in accordance with the actual data.

We now use the model to simulate artificial data, over which we replicate the different structural VARs used in the relevant literature and in the empirical part of the paper. To compute artificial time-series of the variables of interest, we draw $S=1000$ independent random realizations of the TFP innovation $\eta_{z t}$, the investment-specific technology innovation $\eta_{v t}$ and the measurement error innovation $\eta_{c t}$. Using the parameters of Table 2, we compute $S=1000$ equilibrium paths for the growth rates of labor productivity and hours worked. In all experiments, the sample size is equal to 240 quarters, as in actual data. In order to reduce the influence of initial conditions, the simulated sample includes 250 initial points which are subsequently discarded before the estimation of VAR models. For each draw, the number of lags in VAR models is set to 4, a value typically used in empirical studies. The results are reported in Figures 6-9.

Let us first consider the SVAR models that include labor productivity and hours (see the top panel of Figures 6 and 7). Our main results are the following. First, the response of hours obtained from the DSVAR model displays a downward bias when the measure of hours is contaminated and this bias lasts in long-run (not reported in this Figure to save space). This bias decreases when the low frequency movements are removed from hours. Second, the measurement of hours (contaminated and uncontaminated) in the LSVAR model does not affect the estimated response (the LSVAR over-estimates the true response). Third, the confidence interval is smaller and decreases as the horizon of the response increases when uncontaminated hours are used instead 
the contaminated ones. Obviously, uncontaminated hours are less volatile than the contaminated ones, since the latter includes measurement errors. However, we redo the same exercise adjusting for the standard errors of the technology and shock investment shock, such that the volatility of uncontaminated hours equals the one of contaminated hours. We then include this new series in the LSVAR model and compare the response to the LSVAR with contaminated hours, as reported in the top panel of Figure 6. We obtain a large confidence interval for the estimated response, but a decrease with the horizon of the response. This is in contrast with the case of contaminated hours; for which the confidence interval does not decrease with the horizon. We have also investigated the case of stationary measurement errors (we set $c=0$ ). We adjust the volatility of the measurement error shock (to get similar variance of hours) and then compute contaminated hours. Again, we obtain a confidence interval that decreases when the horizon decreases. All these findings echoes our analytical results.

We now consider the SVAR models that include TFP and hours (see the bottom panel of Figures 6 and 7). Now, the first variable which is used to identify a technology shock is not polluted by the low-frequency movement in the contaminated hours. In this case (as shown in the Proposition 1), the low-frequency movements in contaminated hours only affect the long-run variance of hours worked. The figures show that when the econometrician uses a proper measure of the technology, the specification of hours in the VAR model does not matter. Each SVAR consistently estimates the dynamic effects of technology shocks on hours. At the same time, the previous results apply: we obtain a smaller confidence interval if uncontaminated hours are included in the VAR model and this confidence interval decreases with the horizon of the response.

Finally, we investigate the dynamic responses of the productivity measures (labor productivity, TFP) to a technology innovation. These are reported in Figures 8 and 9. Following Proposition 1, the DSVAR model using labor productivity with a contaminated hours must lead to overestimate the true long-run response. This is reported in the bottom of the Figure 8. As previously mentioned, two shocks increases permanently the labor productivity (technology shock and measurement error) and this corrupts the long-run identification strategy. Notice that the estimated short-run responses differs significantly from the true ones and the long-run impact is significantly over-estimated as expected (not reported in the Figure but available on request). When these low-frequency movements are removed from hours worked, the DSVAR model delivers dynamics responses close to the true one. The true responses lies now in the confidence interval of the estimated response. The simulation experiments with the LSVAR model does not deliver clear 
cut evidence. It appears that the estimated response is less biased than those obtained from the DSVAR model and the results seems less sensitive to low frequency movements in hours worked. When the productivity measure in the VAR model is now the TFP, SVAR models yield more accurate dynamic responses.

\section{Conclusion}

This paper studies the statistical properties of impulse response functions in SVARs with a highly persistent variable as hours worked and long-run identifying restrictions. We show that the estimated responses from LSVAR and DSVAR models are biased in finite sample if the measure of productivity is contaminated by low frequency movements in hours. However, if the econometrician uses a proper measure of TFP, the bias disappears for the LSVAR and the DSVAR specifications. We also show that the estimators from LSVAR are asymptotically consistent, but display a nonstandard limiting distribution. This explains the large confidence interval for the dynamic responses in the LSVAR setup. Estimation from US data and simulation experiments from a business cycle model with real frictions confirm our theoretical results.

Our findings can serve as useful guideline to improve the reliability of SVAR models with longrun restrictions. First, our theoretical and empirical results suggest that more efforts must be made to obtain proper measures of TFP as done by Basu, Fernald and Kimball (2006) at annual frequency, because including highly persistent variables in SVAR models is less problematic in this case. ${ }^{15}$ Second, our findings show that part of the poor performances of SVARs is due to the high persistence of hours. Some previous papers have tried to deal with this problem. Francis and Ramey (2009) construct a corrected measure of per capita hours worked that adjusts for low frequency movements in government employment, schooling, and the aging of population. Fève and Guay (2010) propose a simple two step method. In a first step, a consistent estimator of the technology shock is obtained with a SVAR excluding hours worked. The response of hours that follows a technology improvement is estimated in a second step using different linear projections on the estimated technology innovations. Interestingly, these three aforementioned papers find that both level and first-difference specifications yield very similar dynamic responses in SVARs, i.e. a short-run decline followed by a positive hump-shaped response of hours.

\footnotetext{
${ }^{15}$ Recently, Fernald (2009) proposes a quarterly version of this technology measure.
} 


\section{References}

Basu, S., Fernald, J. and M. Kimball (2006) "Are Technology Improvements Contractionary?", American Economic Review, 96(5), pp. 1418-1448.

Beaudry, P. and A. Guay (1996) "What Do Interest Rates Reveal about the Functioning of Real Business Cycle Models?", Journal of Economic Dynamics and Control, 20, 1661-1682.

Blanchard, O.J. and D. Quah (1989) "The Dynamic Effects of Aggregate Demand and Supply Disturbances", American Economic Review, 79(4), pp. 655-673.

Boldrin, M., L. J. Christiano and J. D. Fisher (2001) "Habit Persistence, Asset Returns, and the Business Cycle", American Economic Review, 91(1), 149-166,

Chan, N. and C. Wei (1987) "Asymptotic Inference for Nearly Nonstationary AR(1) Process", Annals of Statistics, 15, pp. 1050-1063.

Chang, Y., Doh, T. and Schorfheide, F. (2007) "Non-stationary Hours in a DSGE Model", Journal of Money, Credit and Banking, 39(6), pp. 1357-1373.

Chari, V., Kehoe, P. and E. Mc Grattan (2008) "A Critique of Structural VARs Using Real Business Cycle Theory", Journal of Monetary Economics, 55, pp. 1337-1352.

Christiano, L. J., M. Eichenbaum and C. L. Evans (2005) "Nominal Rigidities and the Dynamic Effects of a Shock to Monetary Policy", Journal of Political Economy, 113(1), 1-45.

Christiano, L., Eichenbaum, M. and R. Vigfusson (2004a) "What Happens after a Technology Shock?", NBER Working Paper Number 9819, revised version 2004.

Christiano, L., Eichenbaum, M. and R. Vigfusson (2004b) "The Response of Hours to a Technology Shock: Evidence Based on Direct Measures of Technology", Journal of European Economic Association, vol. 2, 2-3, pp. 381-395.

Christiano, L., Eichenbaum, M. and R. Vigfusson (2006) "Assessing Structural VARs", NBER Macroeconomics Annual 2006, Volume 21. D. Acemoglu, K. Rogoff and M. Woodford (Eds)

Erceg, C., Guerrieri, L. and C. Gust (2005) "Can Long-Run Restrictions Identify Technology Shocks", Journal of European Economic Association, 3, pp. 1237-1278.

Fernald, J. (2009) "A Quarterly, Utilization-Adjusted Series on Total Factor Productivity", preliminary draft, Federal Reserve Bank of San Francisco.

Fève, P. and A. Guay (2009) "The Response of Hours to A Technology Shock: A Two-Step Structural VAR Approach", Journal of Money, Credit and Banking, 41(5), pp. 987-1013.

Fève, P. and A. Guay (2010) "Identification of Technology Shocks in Structural VARS", Economic Journal, 120(549), pp. 1284-1318

Francis, N. and V. Ramey (2005) "Is the Technology-Driven Real Business Cycle Hypothesis Dead? Shocks and Aggregate Fluctuations Revisited", Journal of Monetary Economics, 52, pp. 1379-1399.

Francis, N., and V. Ramey (2009) "Measures of per Capita Hours and their Implications for the Technology-Hours Debate", Journal of Money, Credit and Banking, 41(6), pp. 1071-1097. 
Galí, J. (1999) "Technology, Employment and the Business Cycle: Do Technology Shocks Explain Aggregate Fluctuations?", American Economic Review, 89(1), pp. 249-271.

Galí, J., and P. Rabanal (2004) "Technology Shocks and Aggregate Fluctuations; How Well does the RBC Model Fit Postwar U.S. Data?", NBER Macroeconomics Annual, pp. 225-288.

Gospodinov, N. (2010) "Inference in Nearly Nonstationary SVAR Models with Long-Run Identifying Restriction", Journal of Business 86 Economic Statistics, 28(1), pp. 1-12.

Gospodinov, N., A. Maynard and E. Pesavento (2011) "Sensitivity of Impulse Response to Small Low Frequency Co-Movements: Reconciling the Evidence on the Effects of Technology Shocks", Journal of Business 85 Economic Statistics, 29(4), pp. 455-467.

Hamilton, J. (1994) Time Series Analysis, Princeton University Press.

Lewis, R. and G.C. Reinsel (1985) "Prediction of Multivariate Time Series by Autoregressive Model Fitting", Journal of Multivariate Analysis, 15(1), pp. 393-411.

Marcet, A. (2005) "Overdifferencing VAR's is Ok", manuscript.

Ng, S. and P. Perron (2001) "Lag Length Selection and the Construction of Unit Root Tests with Good Size and Power", Econometrica, 69(6), pp. 1519-1554.

Pantula, S. (1991) "Asymptotic Distributions of Unit-Root Tests when the Process is Nearly Stationary", Journal of Business 85 Economic Statistics, 9(1), pp. 63-71.

Perron, P. and S. Ng (1996) "Useful Modifications to some Unit Root Tests with Dependant Errors and their Local Asymptotic Properties", Review of Economic Studies, 63, pp. 435-463.

Phillips, P.C.B. (1987) "Towards a Unified Asymptotic Theory for Autoregression", Biometrika, 74(3), pp. 535-547.

Vigfusson R. (2004) "The Delayed Response to a Technology Shock. A Flexible Price Explanation", International Finance Discussion Paper Series 2004-810. Washington: Board of Governors of the Federal Reserve System, July.

Shapiro, M. and M. Watson (1988) "Sources of Business Cycles Fluctuations", NBER Macroeconomics Annual, Vol. 3, pp. 111-156, S. Fischer Ed. , MIT Press.

Smets, Frank and Rafael Wouters (2007) "Shocks and Frictions in US Business Cycles: A Bayesian DSGE Approach"”, American Economic Review, 97(3), 587-605. 


\section{Appendix}

\section{A Log-Linear Solution of the Model}

The optimality and equilibrium conditions are given by:

$$
\begin{aligned}
\frac{1}{C_{t}} & =\beta\left[1-\delta+\theta \frac{Y_{t+1}}{K_{t+1}}\right] \frac{1}{C_{t+1}} \\
B H_{t} & =(1-\theta) \frac{Y_{t}}{C_{t}} \\
K_{t+1} & =(1-\delta) K_{t}+K_{t}^{\theta}\left(Z_{t} H_{t}\right)^{1-\theta}-C_{t} \\
\Delta \log \left(Z_{t}\right) & =\left(\gamma_{z}-1\right)+\sigma_{z} \varepsilon_{z, t} .
\end{aligned}
$$

In this model, the technology shock $Z_{t}$ induces a stochastic trend into output, consumption, investment and capital. Accordingly, to obtain a stationary equilibrium, these variables must be de-trended as follows

$$
\breve{y}_{t}=\frac{Y_{t}}{Z_{t}}, \quad \breve{c}_{t}=\frac{C_{t}}{Z_{t}}, \quad \breve{i}_{t}=\frac{I_{t}}{Z_{t}}, \quad \breve{k}_{t+1}=\frac{K_{t+1}}{Z_{t}} .
$$

The $\log$-linearization of equilibrium conditions around the deterministic steady state yields

$$
\begin{aligned}
\widehat{\breve{k}}_{t+1} & =\frac{(1-\delta)}{\gamma_{z}}\left(\widehat{\breve{k}}_{t}-\sigma_{z} \eta_{z t}\right)+\frac{y}{k} \widehat{\breve{y}}_{t}-\frac{c}{k} \widehat{c}_{t} \\
\widehat{h}_{t} & =\widehat{\breve{y}}_{t}-\widehat{\breve{c}}_{t} \\
\widehat{\breve{y}}_{t} & =\theta\left(\widehat{\breve{k}}_{t}-\sigma_{z} \eta_{z t}\right)+(1-\theta) \widehat{\breve{h}}_{t} \\
E_{t} \widehat{\breve{c}}_{t+1} & =\widehat{\breve{c}}_{t}+\alpha \beta \frac{y}{k} E_{t}\left(\widehat{\breve{y}}_{t+1}-\widehat{\breve{k}}_{t+1}-\sigma_{z} \eta_{z t+1}\right),
\end{aligned}
$$

where $y / k=\left(\gamma_{z}-\beta(1-\delta)\right) /\left(\theta \beta \gamma_{z}\right)$ and $c / k=y / k-\left(\gamma_{z}+\delta-1\right) / \gamma_{z}$. After substitution of $(23)$ into (24), one gets

$$
\widehat{\breve{y}}_{t}-\widehat{\breve{k}}_{t}=-\sigma_{z} \eta_{z t}-\frac{1-\theta}{\theta} \widehat{\breve{c}}_{t}
$$

Now, using the above expression, equations (22) and (25) rewrite

$$
\begin{aligned}
E_{t} \widehat{\breve{c}}_{t+1} & =\varphi \widehat{\breve{c}}_{t} \quad \text { with } \quad \varphi=\frac{\theta \gamma_{z}}{\gamma_{z}-\beta(1-\theta)(1-\delta)} \in(0,1) \\
\widehat{\breve{k}}_{t+1} & =\nu_{1} \widehat{\breve{k}}_{t}-\nu_{1} \sigma_{z} \eta_{z t}-\nu_{2} \widehat{\breve{c}}_{t} \\
& \quad \text { with } \nu_{1}=\frac{1}{\beta \varphi}>1 \quad \text { and } \quad \nu_{2}=\frac{\gamma_{z}\left(1-\beta \theta^{2}\right)-\beta(1-\delta)\left(1-\theta^{2}\right)}{\theta^{2} \beta \gamma_{z}}
\end{aligned}
$$

As $\nu_{1}>1,(27)$ must be solved forward

$$
\widehat{\breve{k}}_{t}=\sigma_{z} \eta_{z t}+\left(\frac{\nu_{2}}{\nu_{1}}\right) \lim _{T \rightarrow \infty} E_{t} \sum_{i=0}^{T}\left(\frac{1}{\nu_{1}}\right)^{i} \widehat{\breve{c}}_{t+i}+\lim _{T \rightarrow \infty} E_{t}\left(\frac{1}{\nu_{1}}\right)^{T} \widehat{\breve{k}}_{t+T}
$$

Excluding explosive pathes, i.e. $\lim _{T \rightarrow \infty} E_{t}\left(1 / \nu_{1}\right)^{T} \widehat{\breve{k}}_{t+T}=0$, and using (26), one gets the decision rule on consumption:

$$
\widehat{\breve{c}}_{t}=\left(\frac{\nu_{1}-\varphi}{\nu_{2}}\right)\left(\widehat{\breve{k}}_{t}-\sigma_{z} \eta_{z t}\right)
$$


After substituting (28) into (27), the dynamics of capital is given by:

$$
\widehat{\breve{k}}_{t+1}=\varphi\left(\widehat{\breve{k}}_{t}-\sigma_{z} \eta_{z t}\right)
$$

The persistence properties of the model is thus governed by the parameter $\varphi \in(0,1)$, which corresponds to the stable root of the log-linear version of the model. The decision rules of the other (deflated) variables are similar to equation (28). The hours worked are given by

$$
\begin{aligned}
\widehat{h}_{t} & =\widehat{\breve{y}}_{t}-\widehat{\breve{c}}_{t} \\
& =-\nu\left(\widehat{\breve{k}}_{t}-\sigma_{z} \eta_{z t}\right) \\
& =-\nu\left(-\frac{\varphi}{1-\varphi L} \sigma_{z} \varepsilon_{z, t-1}-\sigma_{z} \eta_{z t}\right) \\
& =\nu\left(\frac{\sigma_{z} \eta_{z t}}{1-\varphi L}\right)
\end{aligned}
$$

where $\nu=\alpha\left(\nu_{1}-\varphi-\theta \nu_{2}\right) /\left(\theta \nu_{2}\right)$ is a positive parameter. The latter expression shows that hours worked (or the consumption to output ratio) follows exactly the same stochastic process (an autoregressive process of order one) as the deflated capital $\log \left(K_{t} / Z_{t-1}\right)$ in equation (29).

Using the expression for the growth rate of output

$$
\Delta y_{t}=\widehat{\breve{y}}_{t}-\widehat{\breve{y}}_{t-1}+\sigma_{z} \eta_{z t}
$$

we deduce

$$
\Delta y_{t}=\sigma_{z} \eta_{z t}-\mu \frac{\sigma_{z} \Delta \eta_{z t}}{1-\varphi L}
$$

where $\mu=1-(1-\theta)\left(\nu_{1}-\varphi\right) /\left(\alpha \nu_{2}\right)$.

\section{B Proofs}

Lemma 1 Under the assumptions that $\sum_{i=0}^{\infty} i\left|a_{k j}^{(i)}\right|<\infty$ for $k, j=1,2$ and that $\left\{\eta_{t}\right\}$ is a two dimensional i.i.d. sequence of structural shocks with zero mean, finite fourth moments and $E\left(\eta_{t} \eta_{t}^{\prime}\right)=I_{2}$, we get by Proposition 18.1 in Hamilton (1994, p.548)

$$
\begin{aligned}
& \frac{1}{T} \sum_{t=2}^{T}\left(c \sum_{i=2}^{t} \eta_{2 i-1}\right) \eta_{1 t} \stackrel{L}{\longrightarrow} c \int_{0}^{1} W_{2}(r) d W_{1}(r) \\
& \frac{1}{T} \sum_{t=2}^{T}\left(c \sum_{i=2}^{t} \eta_{2 i-1}\right) \eta_{2 t} \stackrel{L}{\longrightarrow} c \int_{0}^{1} W_{2}(r) d W_{2}(r) \\
& \frac{1}{\sqrt{T}} \sum_{t=2}^{T} a_{k j}(L) \eta_{j t-1} \eta_{l t} \stackrel{L}{\longrightarrow} \mathcal{N}\left(0, \sum_{i=0}^{\infty}\left(a_{k j}^{(i)}\right)^{2}\right)
\end{aligned}
$$

where $W_{1}(r)$ and $W_{2}(r)$ are two standardized independent Brownian motions $l=1,2$. 


\section{B.1 Proof of Theorem 1}

Let us first give the asymptotic variance of $X_{2 t}$ with $c>0$ and $c=0$. For the case where $c>0$, we can show that

$$
\psi_{2}=\lim _{T \rightarrow \infty} \frac{1}{T} \sum_{t=2}^{T} X_{2 t-1} X_{2 t-1}=\sum_{i=0}^{\infty} a_{21}^{2}+\sum_{i=0}^{\infty} a_{22}^{2}+a_{22}(1)^{2} c^{2} .
$$

which depends on the parameter $c$. We also define $\psi_{2,0}$ as the asymptotic variance of $X_{2 t}$ but with $c=0$.

Under the structural model (3) or (4), the numerator of the IV estimator of $b_{12}^{(0)}$ is given by ${ }^{16}$

$$
\frac{1}{T} \sum_{t=2}^{T} X_{2 t-1} \eta_{1 t}=\frac{1}{T} \sum_{t=2}^{T}\left(a_{21}(L) \eta_{1 t-1}+a_{22}(1) \frac{c}{\sqrt{T}} \sum_{j=2}^{t} \eta_{2 j-1}+\tilde{a}_{22, T}^{*}(L) \eta_{2 t-1}\right) \eta_{1 t}
$$

where the partial sum verify

$$
a_{22}(1) \frac{c}{T^{1+1 / 2}} \underbrace{\sum_{t=2}^{T}\left(\sum_{j=2}^{t} \eta_{2 j-1}\right) \eta_{1 t}}_{O_{p}(T)} \stackrel{p}{\rightarrow} 0 .
$$

Asymptotically $\tilde{a}_{22, T}^{*}(L)=a_{22}(L)$ and by Lemma 1 this yields

$$
\frac{1}{T} \sum_{t=2}^{T} a_{21}(L) \eta_{1 t-1} \eta_{1 t} \stackrel{p}{\rightarrow} 0 \quad \text { and } \quad \frac{1}{T} \sum_{t=2}^{T} \tilde{a}_{22 t}^{*}(L) \eta_{2 t-1} \eta_{1 t} \stackrel{p}{\rightarrow} 0
$$

which implies

$$
\frac{1}{T} \sum_{t=2}^{T} X_{2 t-1} \eta_{1 t} \stackrel{p}{\rightarrow} 0
$$

Let us now examine the denominator. By inverting eq. (14) and (15) and using $B_{0}^{-1}=A_{0}$, we get

$$
\begin{aligned}
\Delta \widetilde{X}_{1 t} & =a_{12}^{(0)} b_{22}^{*} \widetilde{X}_{2 t-1}+a_{11}^{(0)} \eta_{1 t}+a_{12}^{(0)} \eta_{2 t} \\
\Delta \widetilde{X}_{2 t} & =a_{22}^{(0)} b_{22}^{*} \widetilde{X}_{2 t-1}+a_{21}^{(0)} \eta_{1 t}+a_{22}^{(0)} \eta_{2 t} .
\end{aligned}
$$

This yields

$$
\begin{aligned}
\frac{1}{T} \sum_{t=2}^{T} X_{2 t-1} \widetilde{\Delta} X_{2 t} & =\frac{1}{T} \sum_{t=2}^{T} X_{2 t-1} \Delta \widetilde{X}_{2 t} \\
& =\frac{1}{T} \sum_{t=2}^{T} X_{2 t-1}\left(a_{22}^{(0)} b_{22}^{*} \widetilde{X}_{2 t-1}+a_{21}^{(0)} \eta_{1 t}+a_{22}^{(0)} \eta_{2 t}\right) \stackrel{p}{\rightarrow} a_{22}^{(0)} b_{22}^{*} \widetilde{\psi}_{2}
\end{aligned}
$$

where $\widetilde{\psi}_{2}=\lim _{T \rightarrow \infty} \frac{1}{T} \sum_{t=2}^{T} \widetilde{X}_{2 t-1} \widetilde{X}_{2 t-1}$. Since $X_{2 t}$ is asymptotically second order stationary and $\widetilde{\psi}_{2} \leq \psi_{2}$ by the projection properties, $\widetilde{\psi}_{2}$ is then bounded. By combining (32) and (35), we get the result that $\widehat{b}_{12}^{(0)}-b_{12}^{(0)} \stackrel{p}{\rightarrow} 0$.

\footnotetext{
${ }^{16}$ To simplify, we suppose here that the initial values are zero.
} 
Now we establish the convergence in distribution of $b_{12}^{(0)}$. Thus

$$
\sqrt{T}\left(\widehat{b}_{12}^{(0)}-b_{12}^{(0)}\right)=\sqrt{T}\left[\frac{\frac{1}{T} \sum_{t=2}^{T} X_{2 t-1} \eta_{1 t}}{\frac{1}{T} \sum_{t=2}^{T} X_{2 t-1} \Delta \widetilde{X}_{2 t}}\right] .
$$

For the numerator

$$
\frac{1}{\sqrt{T}} \sum_{t=2}^{T} X_{2 t-1} \eta_{1 t}=\frac{1}{\sqrt{T}} \sum_{t=2}^{T}\left[a_{21}(L) \eta_{1 t-1}+a_{22}(1) \frac{c}{\sqrt{T}} \sum_{j=2}^{t} \eta_{2 j-1}+\tilde{a}_{22, T}^{*}(L) \eta_{2 t-1}\right] \eta_{1 t} .
$$

By using Lemma 1, eq. (35) and noting that $\tilde{a}_{22, T}^{*}(L) \rightarrow a_{22}(L)$, we deduce

$$
\sqrt{T}\left(\widehat{b}_{12}^{(0)}-b_{12}^{(0)}\right) \stackrel{L}{\longrightarrow} \frac{1}{a_{22}^{(0)} b_{22}^{*} \widetilde{\psi}_{2}}\left[a_{22}(1) c \int_{0}^{1} W_{2}(r) d W_{1}(r)+\psi_{2,0}^{1 / 2} \xi_{1}\right]
$$

where $\xi_{1}$ is the normal distribution $\mathcal{N}(0,1)$.

Consider now the estimator $\widehat{\beta}$

$$
\widehat{\beta}=\left[\frac{1}{T} \sum_{t=2}^{T} z_{t} x_{t}^{\prime}\right]^{-1}\left[\frac{1}{T} \sum_{t=2}^{T} z_{t} \Delta \widetilde{X}_{2 t}\right]=\left[\frac{1}{T} \sum_{t=2}^{T} z_{t} x_{t}^{\prime}\right]^{-1}\left[\frac{1}{T} \sum_{t=2}^{T} z_{t}\left(x_{t}^{\prime} \beta+\eta_{2 t}\right)\right] .
$$

This yields

$$
\widehat{\beta}-\beta=\left[\frac{1}{T} \sum_{t=2}^{T} z_{t} x_{t}^{\prime}\right]^{-1}\left[\frac{1}{T} \sum_{t=2}^{T} z_{t} \eta_{2 t}\right]
$$

where more explicitly

$$
\begin{aligned}
\frac{1}{T} \sum_{t=2}^{T} z_{t} x_{t}^{\prime} & =\left[\begin{array}{cc}
\frac{1}{T} \sum_{t=2}^{T} \Delta \widetilde{X}_{1 t}\left[\eta_{1 t}-\left(\widehat{b}_{12}^{(0)}-b_{12}^{(0)}\right) \Delta \widetilde{X}_{2 t}\right] & \frac{1}{T} \sum_{t=2}^{T} \widetilde{X}_{2 t-1}\left[\eta_{1 t}-\left(\widehat{b}_{12}^{(0)}-b_{12}^{(0)}\right) \Delta \widetilde{X}_{2 t}\right] \\
\frac{1}{T} \sum_{t=2}^{T} X_{2 t-1} \Delta \widetilde{X}_{1 t} & \frac{1}{T} \sum_{t=2}^{T} X_{2 t-1} \widetilde{X}_{2 t-1}
\end{array}\right] \\
& =\left[\begin{array}{ll}
G_{11, T} & G_{12, T} \\
G_{21, T} & G_{22, T}
\end{array}\right],
\end{aligned}
$$

and

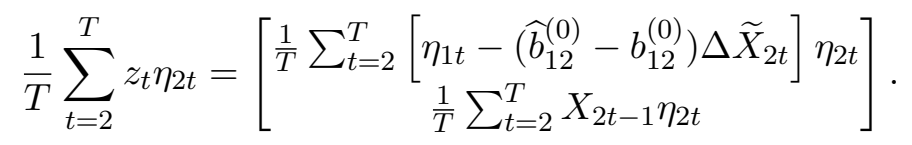

Let us examine the first term $G_{11, T}$,

$$
G_{11, T}=\frac{1}{T} \sum_{t=2}^{T} \Delta \tilde{X}_{1 t} \eta_{1 t}-\left(\widehat{b}_{12}^{(0)}-b_{12}^{(0)}\right) \frac{1}{T} \sum_{t=2}^{T} \Delta \widetilde{X}_{1 t} \Delta \widetilde{X}_{2 t}=\frac{1}{T} \sum_{t=2}^{T} \Delta \widetilde{X}_{1 t} \eta_{1 t}+o_{p}(1)
$$

by $\left(\widehat{b}_{12}^{(0)}-b_{12}^{(0)}\right) \stackrel{p}{\longrightarrow} 0$ and $\frac{1}{T} \sum_{t=2}^{T} \Delta \widetilde{X}_{1 t} \Delta \widetilde{X}_{2 t}=O_{p}(1)$. Using eq. (33), this gives $\frac{1}{T} \sum_{t=2}^{T} \Delta \widetilde{X}_{1 t} \eta_{1 t} \stackrel{p}{\rightarrow}$ $a_{11}^{(0)}$ which implies $G_{11, T} \stackrel{p}{\rightarrow} a_{11}^{(0)}$. The term $G_{21, T}=\frac{1}{T} \sum_{t=2}^{T} X_{2 t-1} \Delta \widetilde{X}_{1 t} \stackrel{p}{\rightarrow} a_{12}^{(0)} b_{22}^{*} \widetilde{\psi}_{2}$ using eq. (33). For the upper right term, we obtain

$$
G_{12, T}=\frac{1}{T} \sum_{t=2}^{T} \widetilde{X}_{2 t-1}\left[\eta_{1 t}-\left(\widehat{b}_{12}^{(0)}-b_{12}^{(0)}\right) \Delta \widetilde{X}_{2 t}\right]=\frac{1}{T} \sum_{t=2}^{T} \widetilde{X}_{2 t-1} \eta_{1 t}+o_{p}(1)
$$


and $\frac{1}{T} \sum_{t=2}^{T} \widetilde{X}_{2 t-1} \eta_{1 t} \stackrel{p}{\rightarrow} 0$ by (32). Finally, $G_{22, T}=\frac{1}{T} \sum_{t=2}^{T} X_{2 t-1} \widetilde{X}_{2 t-1} \stackrel{p}{\longrightarrow} \widetilde{\psi}_{2}$.

Let us examine the expression (36). We have the following results $\frac{1}{T} \sum_{t=2}^{T} \eta_{1 t} \eta_{2 t} \stackrel{p}{\rightarrow} 0,\left(\widehat{b}_{12}^{(0)}-\right.$ $\left.b_{12}^{(0)}\right) \frac{1}{T} \sum_{t=2}^{T} \Delta \widetilde{X}_{2 t} \eta_{2 t} \stackrel{p}{\rightarrow} 0$ and $\frac{1}{T} \sum_{t=2}^{T} X_{2 t-1} \eta_{2 t} \stackrel{p}{\rightarrow} 0$. We can now conclude that $\widehat{\beta}-\beta \stackrel{p}{\longrightarrow} 0$.

To establish the convergence in distribution of $b_{21}^{(0)}$ and $b_{22}^{*}$, we use the following expression $\left.\sqrt{T}\left[\begin{array}{c}\widehat{b}_{21}^{(0)}-b_{21}^{(0)} \\ \widehat{b}_{22}^{*}-b_{22}^{*}\end{array}\right]=\frac{1}{G_{11, T} G_{22, T}-G_{21, T} G_{12, T}}\left[\begin{array}{cc}G_{22, T} & -G_{12, T} \\ -G_{21, T} & G_{11, T}\end{array}\right]\left[\begin{array}{c}\frac{1}{\sqrt{T}} \sum_{t=2}^{T}\left[\eta_{1 t}-\left(\widehat{b}_{12}^{(0)}-b_{12}^{(0)}\right) \Delta \widetilde{X}_{2 t}\right.\end{array}\right] \eta_{2 t}\right]$.

We now examine the first term at the RHS of (37). From the results derived above, we obtain $G_{11, T} G_{22, T} \stackrel{p}{\rightarrow} a_{11}^{(0)} \widetilde{\psi}_{2}$ and $G_{21, T} G_{12, T} \stackrel{p}{\rightarrow} 0$. For the last term of (37), we have $\frac{1}{\sqrt{T}} \sum_{t=2}^{T} \eta_{1 t} \eta_{2 t} \stackrel{L}{\rightarrow} \xi_{2}$ where $\xi_{2} \sim \mathcal{N}(0,1)$ and

$$
\sqrt{T}\left(\widehat{b}_{12}^{(0)}-b_{12}^{(0)}\right) \frac{1}{T} \sum_{t=2}^{T} \Delta \widetilde{X}_{2 t} \eta_{2 t} \stackrel{L}{\longrightarrow} \frac{\psi_{2,0}^{1 / 2}}{b_{22}^{*} \widetilde{\psi}_{2}} \xi_{1}+\frac{\vartheta_{1}}{b_{22}^{*} \widetilde{\psi}_{2}},
$$

since $\frac{1}{T} \sum_{t=2}^{T} \Delta \tilde{X}_{2 t} \eta_{2 t} \stackrel{p}{\rightarrow} a_{22}^{(0)}$ with $\vartheta_{1}=a_{22}(1) c \int_{0}^{1} W_{2}(r) d W_{1}(r)$ derived above. Now for the expression $\frac{1}{\sqrt{T}} \sum_{t=2}^{T} X_{2 t-1} \eta_{2 t}$,

$$
\frac{\sqrt{T}}{T} \sum_{t=2}^{T} X_{2 t-1} \eta_{2 t}=\frac{1}{T} \sum_{t=2}^{T} \sqrt{T}\left[a_{21}(L) \eta_{1 t-1}+a_{22}(1) \frac{c}{\sqrt{T}} \sum_{j=2}^{t} \eta_{2 j-1}+\tilde{a}_{22, T}^{*}(L) \eta_{2 t-1}\right] \eta_{2 t} .
$$

By using Lemma 1,

$$
\frac{\sqrt{T}}{T} \sum_{t=2}^{T} X_{2 t-1} \eta_{2 t} \stackrel{L}{\longrightarrow}\left[a_{22}(1) c \int_{0}^{1} W_{2}(r) d W_{2}(r)+\psi_{2,0}^{1 / 2} \xi_{2}\right] .
$$

By collecting these results, we obtain that

$$
\sqrt{T}\left(\widehat{b}_{21}^{(0)}-b_{21}^{(0)}\right) \stackrel{L}{\longrightarrow} \frac{\xi_{2}}{a_{11}^{(0)}}-\frac{\psi_{2,0}^{1 / 2}}{a_{11}^{(0)} b_{22}^{*} \widetilde{\psi}_{2}} \xi_{1}-\frac{\vartheta_{1}}{a_{11}^{(0)} b_{22}^{*} \widetilde{\psi}_{2}} .
$$

Now for $\widehat{b}_{22}^{*}$, we get

$$
\sqrt{T}\left(\widehat{b}_{22}^{*}-b_{22}^{*}\right) \stackrel{L}{\longrightarrow} \frac{a_{12}^{(0)} \psi_{2,0}^{1 / 2}}{a_{11}^{(0)} \widetilde{\psi}_{2}} \xi_{1}+\frac{a_{12}^{(0)} \vartheta_{1}}{a_{11}^{(0)} \widetilde{\psi}_{2}}-\left[\frac{a_{12}^{(0)} b_{22}^{*}}{a_{11}^{(0)}}-\frac{\psi_{2,0}^{1 / 2}}{\widetilde{\psi}_{2}}\right] \xi_{2}+\frac{\vartheta_{2}}{\widetilde{\psi}_{2}}
$$

where $\vartheta_{2}=a_{22}(1) c \int_{0}^{1} W_{2}(r) d W_{2}$.

\section{B.2 Proof of Proposition 1}

According to the structural representation (4), the finite sample measure of the long-run impact is given by:

$$
A_{T}(1)=\left[\begin{array}{cc}
a_{11}(1) & a_{12}(1) c / \sqrt{T} \\
0 & a_{22}(1) c / \sqrt{T}
\end{array}\right]
$$


This matrix is not lower triangular as imposed in the identification procedure of the SVAR. The corresponding long-run variance-covariance matrix is then:

$$
A_{T}(1) A_{T}(1)^{\prime}=\left[\begin{array}{cc}
a_{11}(1)^{2}+a_{12}(1)^{2} c^{2} / T & a_{12}(1) a_{22}(1) c^{2} / T \\
a_{12}(1) a_{22}(1) c^{2} / T & a_{22}(1)^{2} c^{2} / T
\end{array}\right]
$$

Now, we wrongly impose a lower triangular form using a Choleski decomposing on (38). In that respect, we can rewrite the expression above using equation (4.4.12) in Hamilton (1994 p.90) as

$$
\begin{aligned}
A_{T}(1) A_{T}(1)^{\prime}= & {\left[\begin{array}{cc}
1 & 0 \\
\frac{a_{12}(1) a_{22}(1) c^{2} / T}{a_{11}(1)^{2}+a_{12}(1)^{2} c^{2} / T} & 1
\end{array}\right]\left[\begin{array}{cc}
a_{11}(1)^{2}+a_{12}(1)^{2} c^{2} / T & 0 \\
0 & a_{22}(1)^{2} c^{2} / T-\frac{a_{12}(1)^{2} a_{22}(1)^{2} c^{4} / T^{2}}{a_{11}(1)^{2}+a_{12}(1)^{2} c^{2} / T}
\end{array}\right] } \\
& \times\left[\begin{array}{cc}
1 & \frac{a_{12}(1) a_{22}(1) c^{2} / T}{a_{11}(1)^{2}+a_{12}(1)^{2} c^{2} / T} \\
0 & 1
\end{array}\right] .
\end{aligned}
$$

By a Choleski decomposition for $A_{T}(1) A_{T}(1)^{\prime}$ we obtain the lower triangular matrix:

$$
\operatorname{chol}\left(A_{T}(1) A_{T}(1)^{\prime}\right)=\left[\begin{array}{cc}
\left(a_{11}(1)^{2}+a_{12}(1)^{2} c^{2} / T\right)^{1 / 2} & 0 \\
\frac{a_{12}(1) a_{22}(1) c^{2} / T}{\left(a_{11}(1)^{2}+a_{12}(1)^{2} c^{2} / T\right)^{1 / 2}} & \left(a_{22}(1)^{2} c^{2} / T-\frac{a_{12}(1)^{2} a_{22}(1)^{2} c^{4} / T^{2}}{a_{11}(1)^{2}+a_{12}(1)^{2} c^{2} / T}\right)^{1 / 2}
\end{array}\right] .
$$


Table 1: Long-Run effect of a Technology Improvement on Productivity Measures (in \%)

\begin{tabular}{lcc}
\hline \hline & LSVAR model & DSVAR Model \\
\hline Solow Residual & 1.51 & 1.63 \\
& {$[0.90 ; 2.93]$} & {$[0.86 ; 2.48]$} \\
"Purified" Measure of TFP & 1.33 & 1.39 \\
& {$[0.80 ; 2.18]$} & {$[0.78 ; 2.09]$} \\
Labor Productivity & 2.05 & 2.32 \\
& {$[1.06 ; 3.92]$} & {$[1.10 ; 3.77]$} \\
\hline \hline
\end{tabular}

Notes: 95\% percent confidence interval in brackets obtained from a standard bootstrap technique with 1000 replications.

Table 2: Parameter values $\Psi$

\begin{tabular}{ccccc}
\hline \hline Calibrated $\Psi_{1}$ & \multicolumn{4}{c}{ Estimated $\Psi_{2}$} \\
Parameter & Value & Parameter & Value & s.e. \\
\hline$\beta$ & 0.9950 & $b$ & 0.4063 & 0.0380 \\
$\alpha$ & 0.3300 & $\zeta$ & 23.8476 & 2.6220 \\
$\delta$ & 0.0153 & $\gamma_{z}$ & 1.0035 & 0.0008 \\
$\nu$ & 2.0000 & $\sigma_{z}$ & 0.0128 & 0.0006 \\
& & $\rho_{v}$ & 0.3131 & 0.0659 \\
& & $\sigma_{v}$ & 0.6669 & 0.0743 \\
& $\rho_{c}$ & 0.6893 & 0.1138 \\
& $c$ & 7.4120 & 2.0615 \\
& & $\sigma_{c}$ & 0.0071 & 0.0004 \\
\hline \hline
\end{tabular}

Note: US quarterly data covering the sample period 1948:1-2007:4. The vector of observed data includes the growth rate of the real GDP, real consumption expenditures (non-durable \& services) and hours worked (per capita). 
Figure 1: US Data
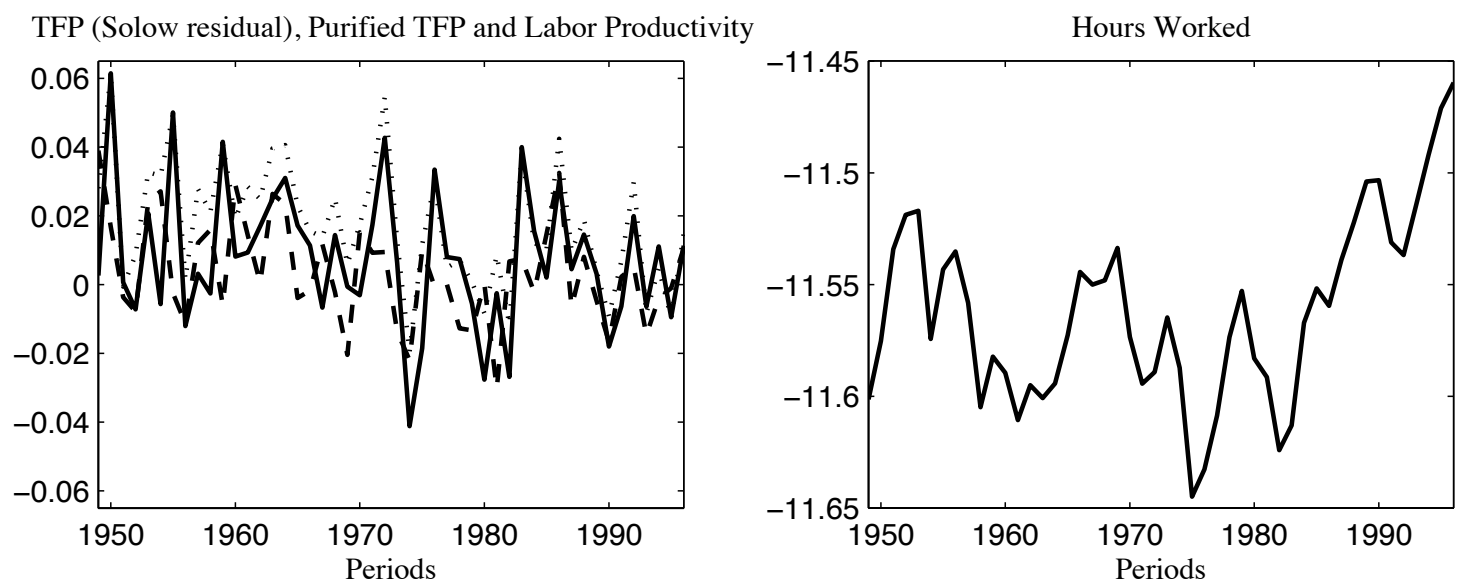

Note: The left hand side of the figure reports different measures of productivity. The solid line refers to the Solow residual, the dashed line to the purified measure of TFP and the dotted line to the labor productivity. All are specified in logs and in first difference. The right hand side reports the log of per capita hours worked. The data are at annual frequency and cover the sample period 1949-1996. 
Figure 2: IRFs of Hours Worked to a Technological Improvement

TFP (Solow residual)
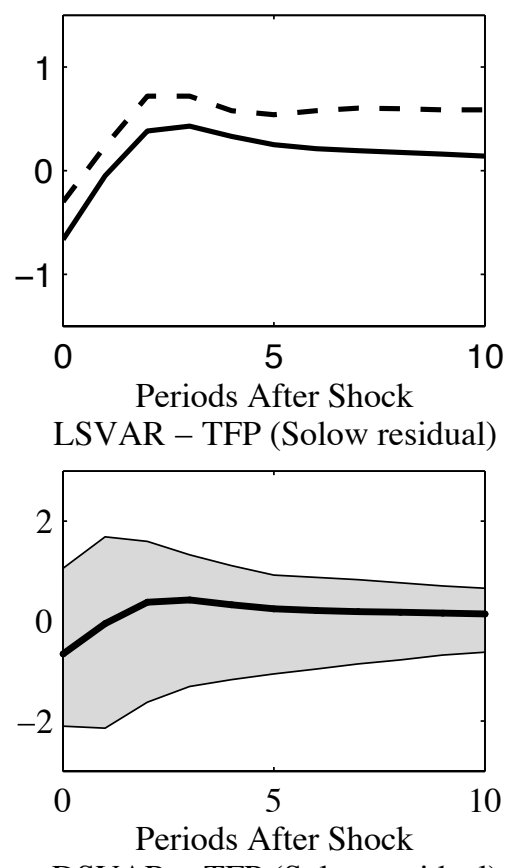

DSVAR - TFP (Solow residual)

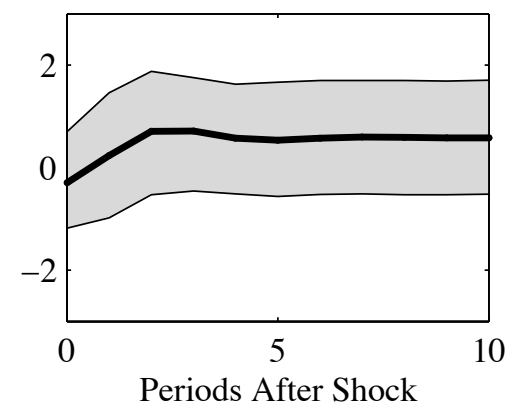

Purified TFP

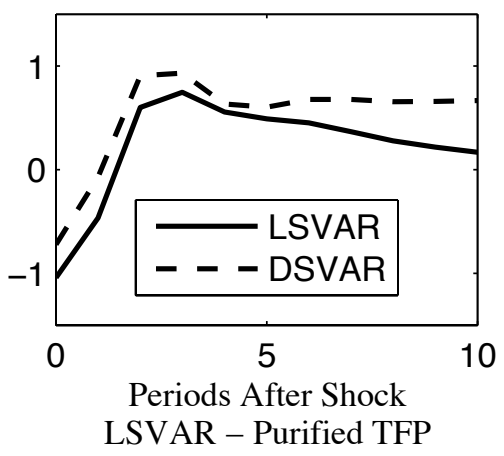

LSVAR - Purified TFP
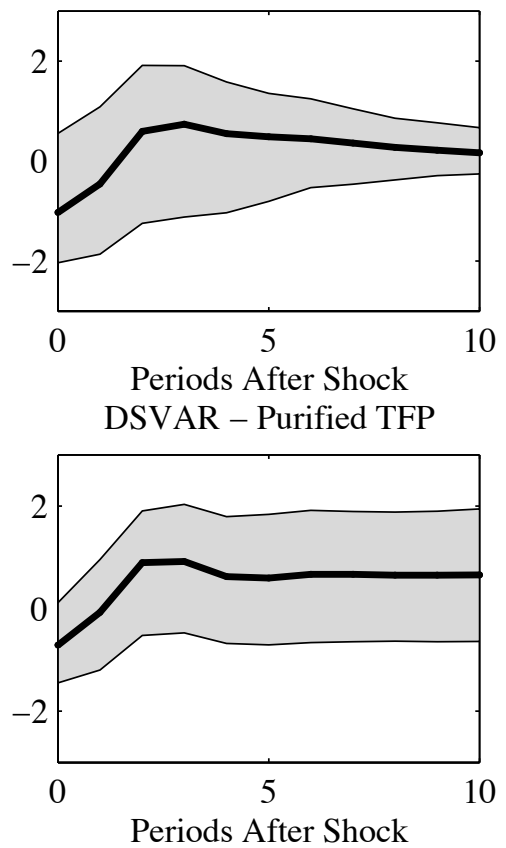

Labor Productivity

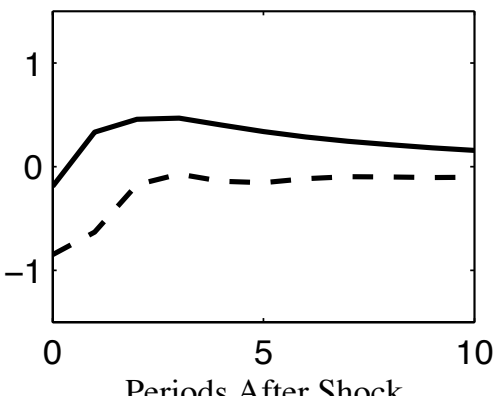

Periods After Shock

LSVAR - Labor Productivity

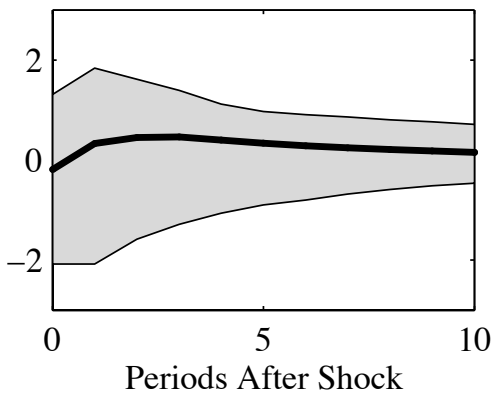
DSVAR - Labor Productivity

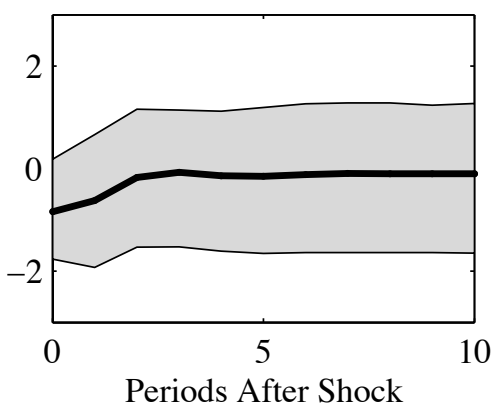

Note: The DSVAR model includes alternatively the growth rate of the Solow residual, the "purified" measure of TFP and the labor productivity, and the log of hours in first difference. The LSVAR model includes alternatively the growth rate of the Solow residual, the "purified" measure of TFP and the labor productivity, and the log of hours in level. The sample period is 1949-1996. Two lags are included in each VAR model. The selected horizon for IRFs is $11.95 \%$ percent confidence interval obtained from a standard bootstrap technique with 1000 replications. 
Figure 3: IRFs of Technology Measures to a Technological Improvement

TFP (Solow residual)

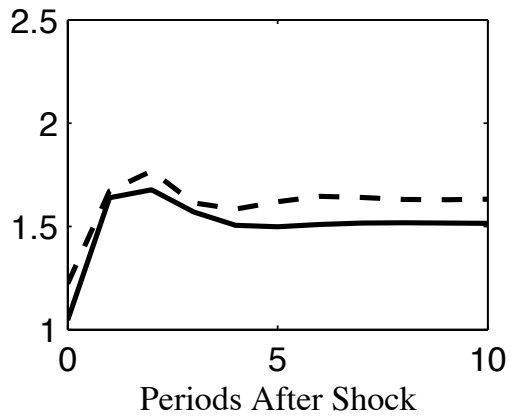

LSVAR - TFP (Solow residual)

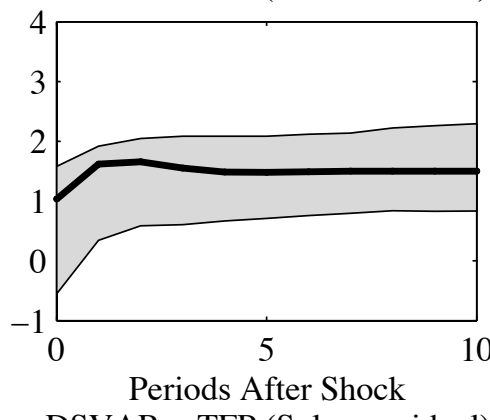

DSVAR - TFP (Solow residual)

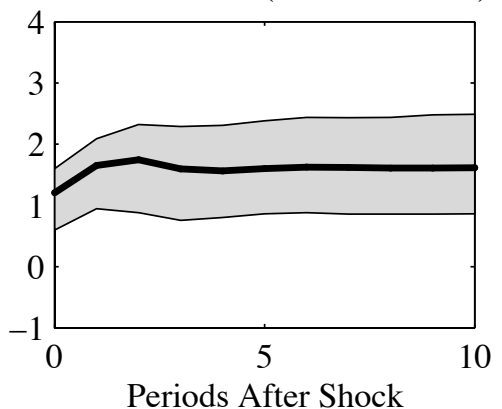

Purified TFP
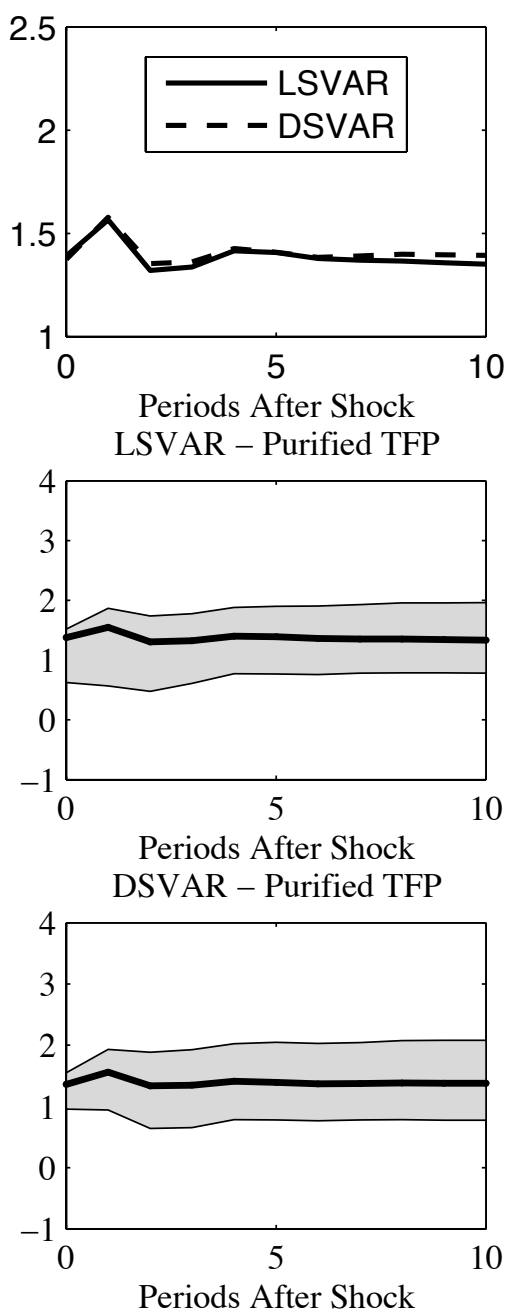

Labor Productivity

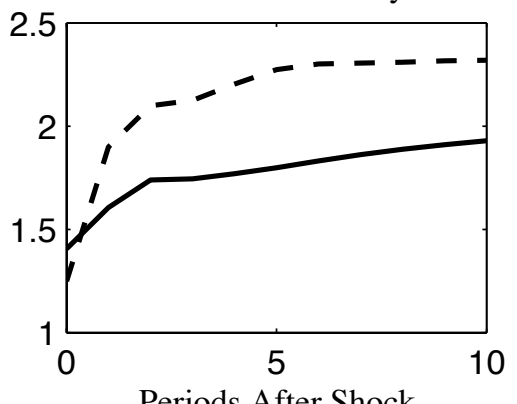

Periods After Shock

LSVAR - Labor Productivity
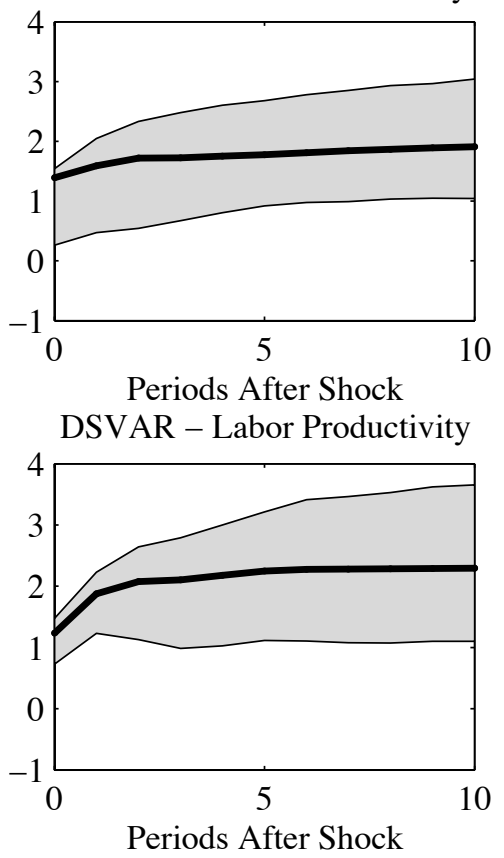

Note: The DSVAR model includes alternatively the growth rate of the Solow residual, the "purified" measure of TFP and the labor productivity, and the log of hours in first difference. The LSVAR model includes alternatively the growth rate of the Solow residual, the "purified" measure of TFP and the labor productivity, and the log of hours in level. The sample period is 1949-1996. Two lags are included in each VAR model. The selected horizon for IRFs is $11.95 \%$ percent confidence interval obtained from a standard bootstrap technique with 1000 replications. 
Figure 4: IRF of Hours

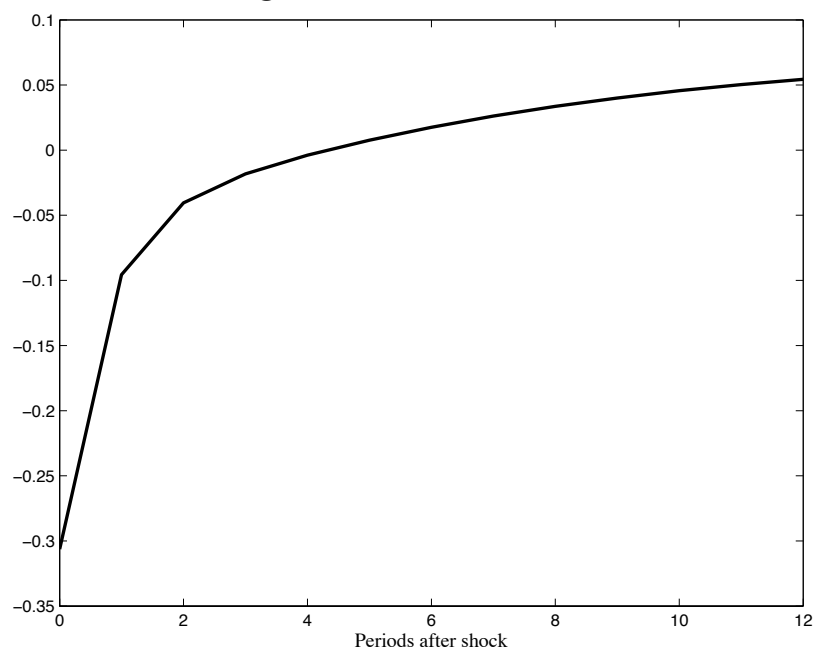

Figure 5: ACFs

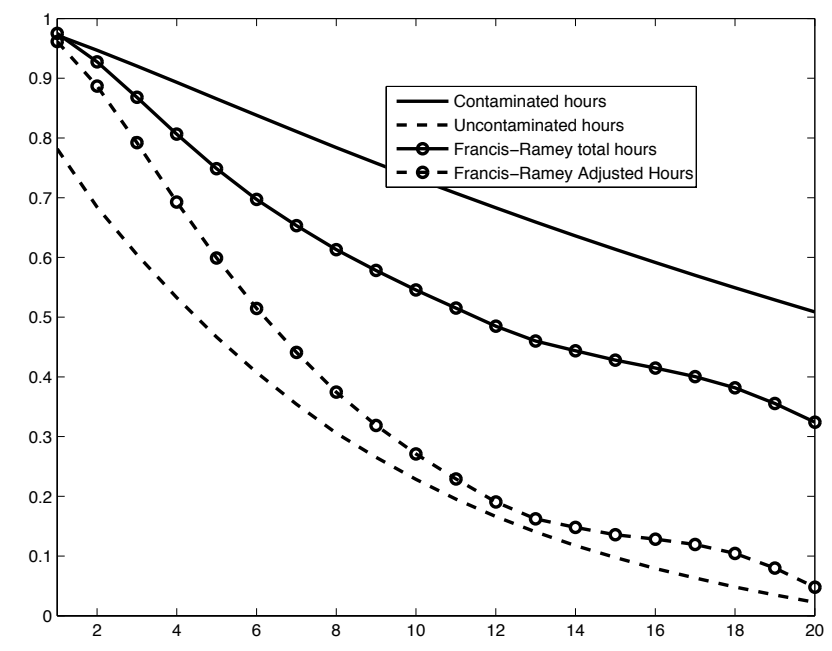




\section{Figure 6: IRF of Hours: Labor Productivity and Hours}

Labor Productivity and Hours
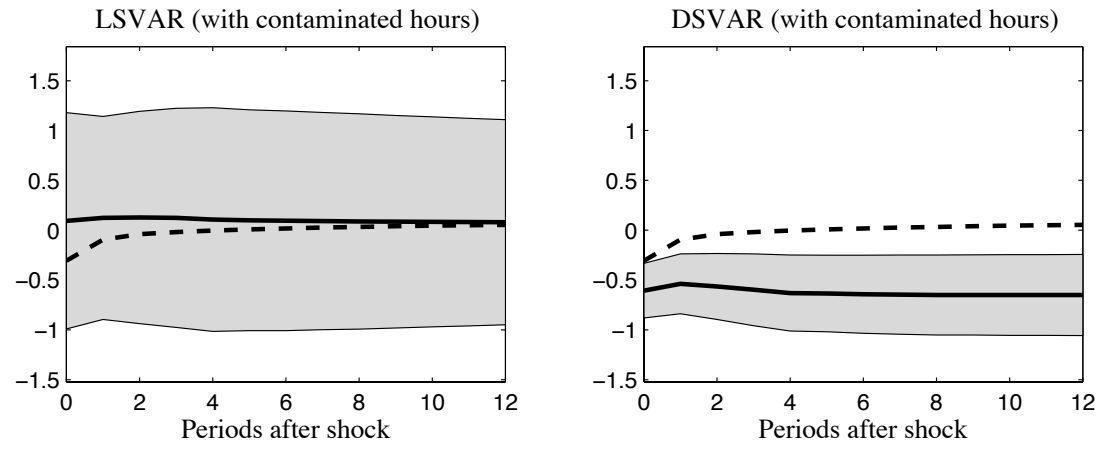

LSVAR (with uncontaminated hours)

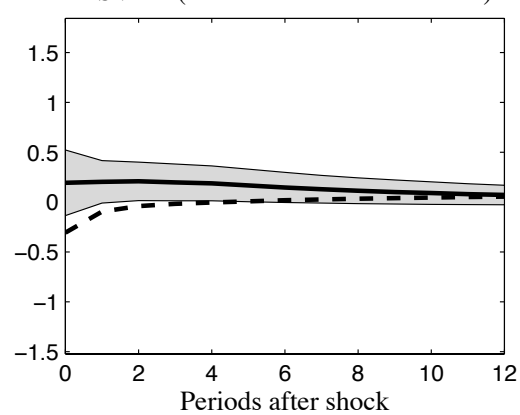

DSVAR (with uncontaminated hours)

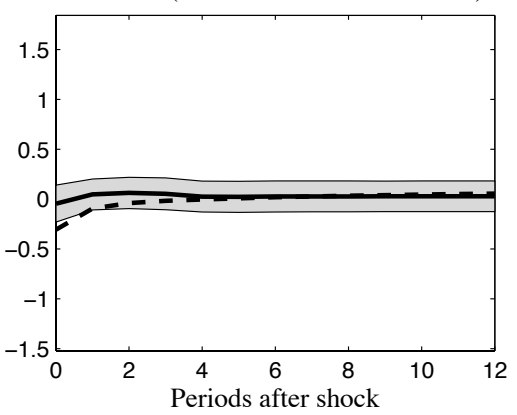

TFP and Hours

LSVAR (TFP with contaminated hours)

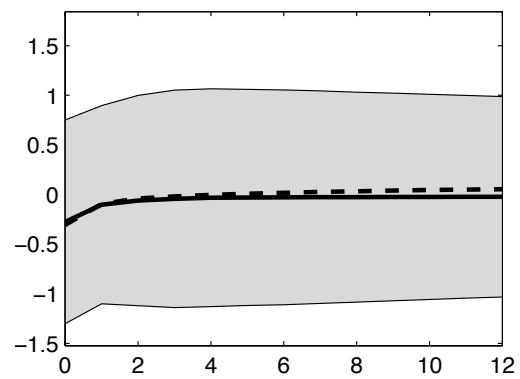

DSVAR (TFP with contaminated hours)

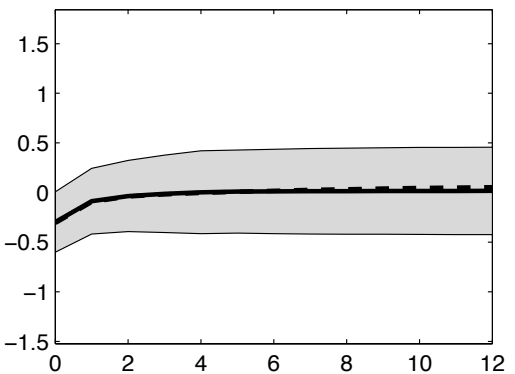

LSVAR (TFP with uncontaminated hours)

DSVAR (TFP with uncontaminated hours)
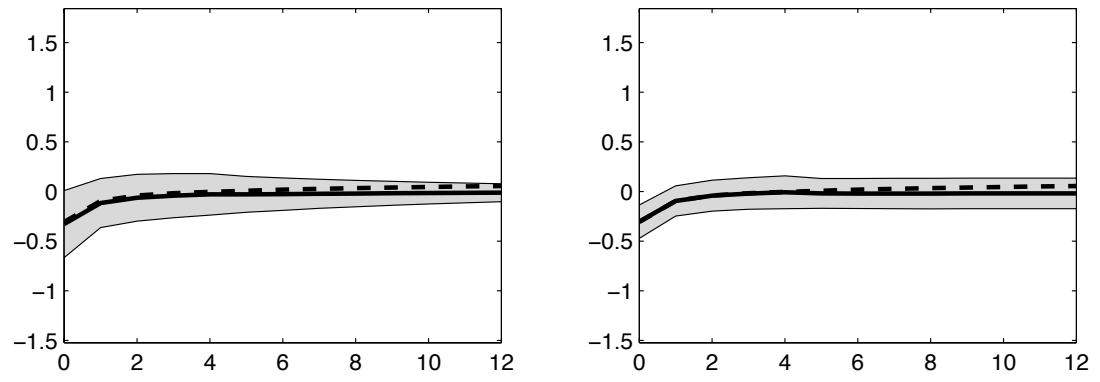

Note: The DSVAR model includes labor productivity growth or TFP growth and the log of hours (contaminated and uncontaminated) in first difference. The LSVAR model includes labor productivity growth or TFP growth and the log of hours (contaminated and uncontaminated). The selected horizon for IRFs is 13. The number of lags in VAR model is $4.95 \%$ confidence interval shown. 
Figure 7: IRF of Hours: Comparison of SVARs
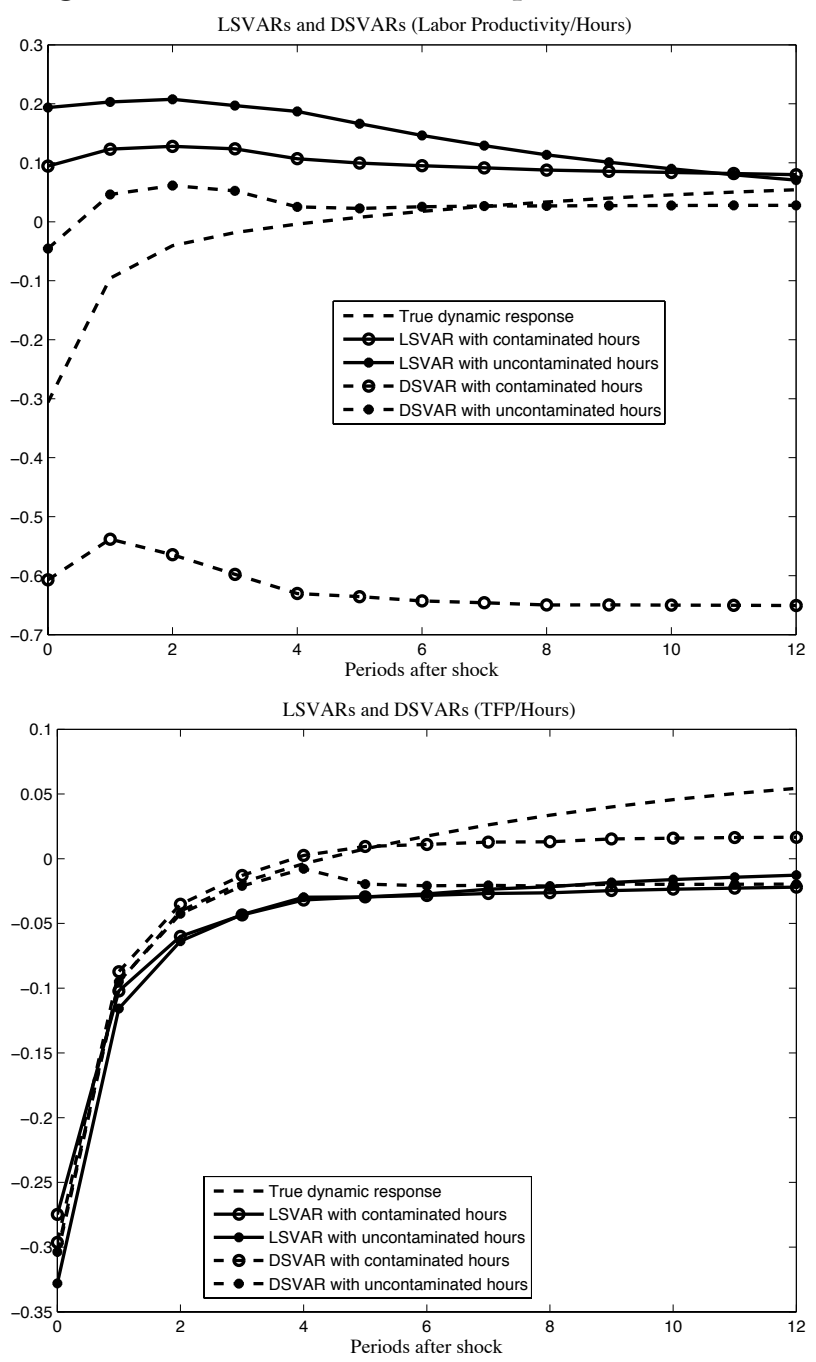

Note: The DSVAR model includes labor productivity growth or TFP growth and the log of hours (contaminated and uncontaminated) in first difference. The LSVAR model includes labor productivity growth or TFP growth and the log of hours (contaminated and uncontaminated). The selected horizon for IRFs is 13. The number of lags in VAR model is 4. 
Figure 8: IRF of Productivity

Labor Productivity and Hours

LSVAR (Labor Productivity with contaminated hourßSSVAR (Labor Productivity with contaminated hours)
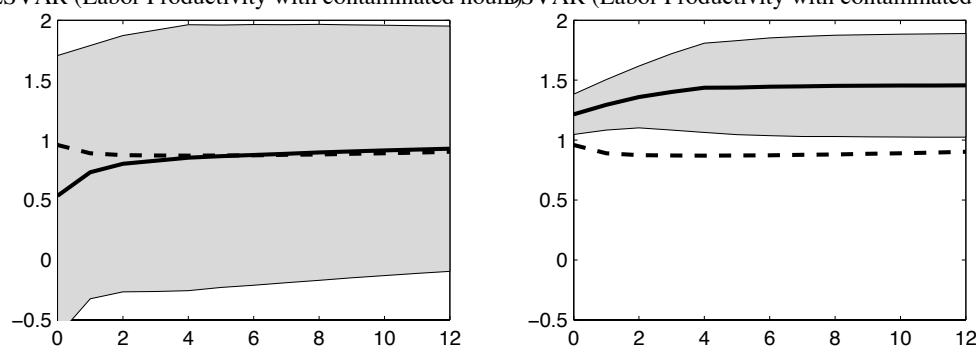

LSVAR (Labor Productivity with uncontaminated hdDSsy AR (Labor Productivity with uncontaminated hours)
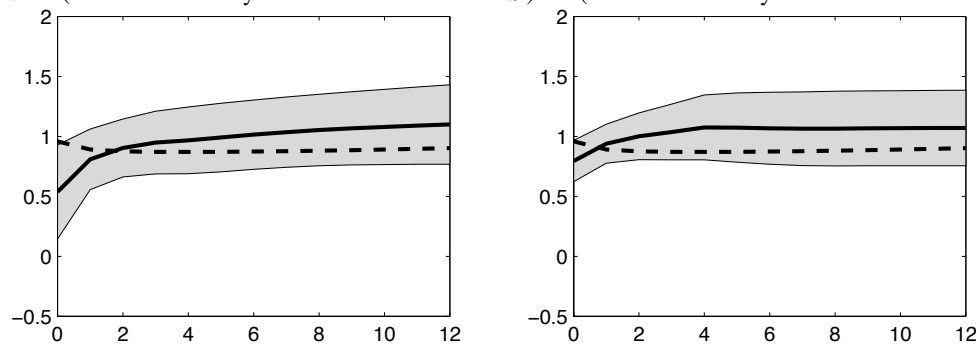

TFP and Hours

LSVAR (TFP with contaminated hours)
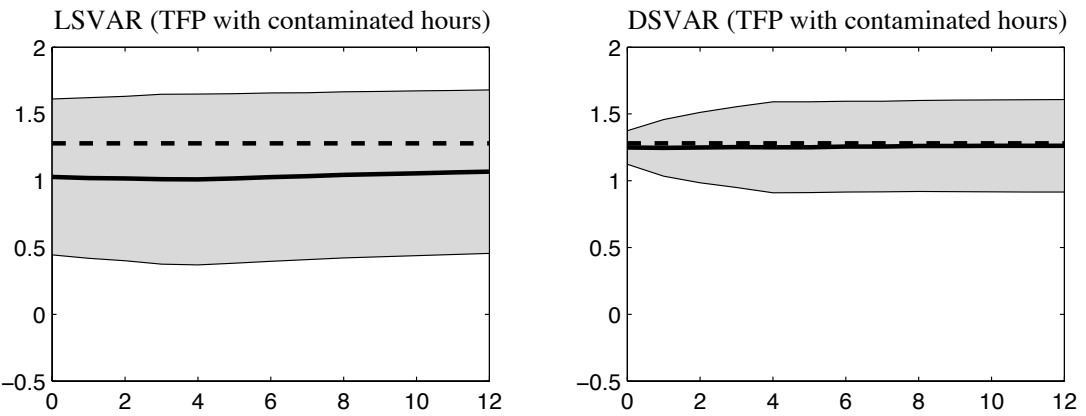

LSVAR (TFP with uncontaminated hours)

DSVAR (TFP with uncontaminated hours)
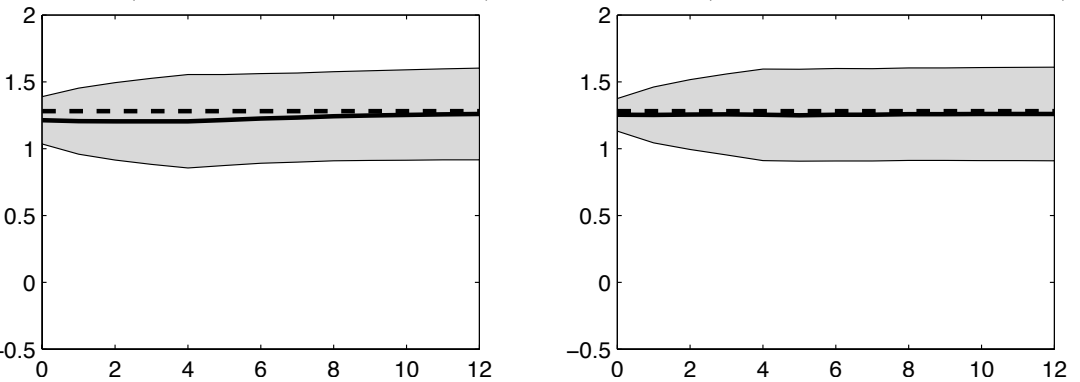

Note: The DSVAR model includes labor productivity growth or TFP growth and the log of hours (contaminated and uncontaminated) in first difference. The LSVAR model includes labor productivity growth or TFP growth and the log of hours (contaminated and uncontaminated). The selected horizon for IRFs is 13. The number of lags in VAR model is $4.95 \%$ confidence interval shown. 
Figure 9: IRF of Productivity Measure: Comparison of SVARs
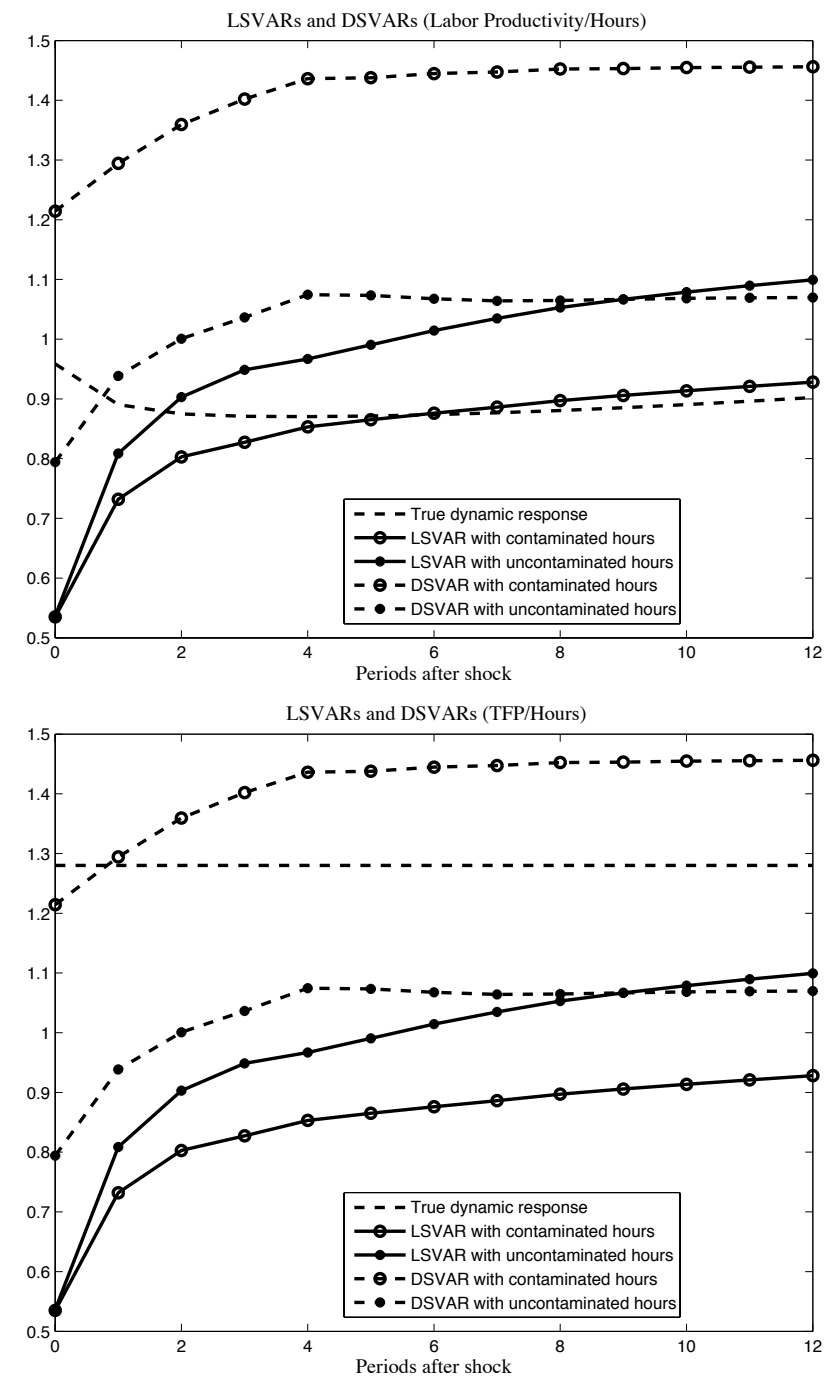

Note: The DSVAR model includes labor productivity growth or TFP growth and the log of hours (contaminated and uncontaminated) in first difference. The LSVAR model includes labor productivity growth or TFP growth and the log of hours (contaminated and uncontaminated). The selected horizon for IRFs is 13 . The number of lags in VAR model is 4 . 